\title{
eJRIEPS
}

Ejournal de la recherche sur l'intervention en éducation physique et sport

$37 \mid 2016$

Varia

\section{L'activité d'enseignants experts en Education Physique et Sportive : une étude de cas sur les formes, les fonctions et la pertinence des communications non verbales en situation}

Jérôme Visioli, Oriane Petiot et Luc Ria

\section{(2) OpenEdition \\ Journals \\ Édition électronique \\ URL : http://journals.openedition.org/ejrieps/1200 \\ DOI : 10.4000/ejrieps. 1200 \\ ISSN : 2105-0821 \\ Éditeur \\ ELLIADD}

Édition imprimée

Date de publication : 1 janvier 2016

\section{Référence électronique}

Jérôme Visioli, Oriane Petiot et Luc Ria, «L'activité d'enseignants experts en Education Physique et Sportive : une étude de cas sur les formes, les fonctions et la pertinence des communications non verbales en situation », eJRIEPS [En ligne], 37 | 2016, mis en ligne le 01 janvier 2016, consulté le 03 octobre 2019. URL : http://journals.openedition.org/ejrieps/1200 ; DOI : 10.4000/ejrieps.1200

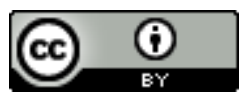

La revue eJRIEPS est mise à disposition selon les termes de la Creative Commons Attribution 4.0 International License. 


\section{eJRIEPS 37 janvier 2016}

\section{L'activité d'enseignants experts en Education Physique et Sportive : une étude de cas sur les formes, les fonctions} et la pertinence des communications non verbales en situation

Jérôme Visioli ${ }^{\star}$, Oriane Petiot** et Luc Ria

*Université Rennes 2, France

**Université Rennes 2, France

*** Université Blaise Pascal Clermont-Ferrand 2, ENS de Lyon, France

\section{Résumé}

L'objectif de cet article est d'analyser l'activité d'enseignants experts en cours d'EPS avec une focale sur les formes, les fonctions et la pertinence de leurs communications non verbales (CNV) en situation. Plus précisément, il s'agit d'une part de mettre en lien les formes et les fonctions des CNV avec les communications verbales (CV), et d'autre part d'évaluer leur pertinence au regard du contexte de classe. En nous appuyant sur le cadre $d u$ "cours d'action " (Theureau, 2006), nous avons analysé l'activité de cinq enseignants durant deux cours d'EPS. Deux principaux types de données ont été recueillis, conduisant à deux types de traitements spécifiques : (1) des données d'observation et de verbalisations des enseignants in situ; (2) des données de verbalisation a posteriori lors d'entretiens d'autoconfrontation. Les résultats présentent l'analyse de l'activité des enseignants, et notamment de leurs CNV, sous la forme de cinq études de cas sélectionnées sur la base des données d'autoconfrontation. Ils sont ensuite discutés selon deux axes : (1) la complexité des relations entre les formes et les fonctions des CNV des enseignants experts en EPS et leurs CV en situation de classe ; (2) les relations entre les CNV et le contexte de classe : la pertinence des CNV.

Mots-clés : activité de l'enseignant, communications non verbales, expertise, contexte de classe, cours d'action

\section{Introduction}

Selon Tardif et Gauthier (2001), dans un contexte d'évolution des caractéristiques des publics scolaires, "l'enseignant doit attacher suffisamment de prix à l'adhésion du groupe pour produire son discours. Cela implique un certain arsenal de compétences de la part de l'enseignant: théâtralisation, capacité de jouer de son autorité, habiletés de 


\section{eJRIEPS 37 janvier 2016}

communication, etc. » (p. 236). Les recherches en sciences de l'éducation ont en effet montré que la communication, notamment non verbale, participaient d'un savoir-être auprès des élèves (Runtz-Christian, 2000), d'une qualité de présence (Lisahunter, 2011), mais aussi d'une amélioration des apprentissages des élèves (Forest, 2008). En particulier, les enseignants experts semblent développer des compétences à jouer avec l'expression de leur corps pour obtenir des effets variés sur les élèves (Visioli, Petiot \& Ria, 2014). A l'inverse, selon Moulin (2004), un certain nombre d'enseignants novices connaissent des problèmes de communication, liés notamment à la relation d'autorité, qu'ils passent une grande partie du temps à gérer et qui absorbe leur énergie. Etudier les communications non verbales (CNV) des experts, constitutives de l'agir enseignant (Bucheton \& Soulé, 2009), apparaît dès lors porteurs d'enjeux professionnels majeurs.

Plus généralement, Winkin (1981) a proposé de regrouper sous le terme de "nouvelle communication " un vaste courant regroupant des chercheurs venant d'horizons divers (Bateson, 1984 ; Birdwhistell, 1974 ; Goffman, 1973 ; Hall, 1984 ; Watzlawick, 1988), qui considèrent la communication comme un processus social permanent au sein duquel les CNV jouent un rôle central. Les études sur la communication humaine (Cosnier, 1982) s'accordent à dire que celle-ci est multicanal : l'énoncé verbal partage la signification du message avec d'autres canaux de communication, notamment le canal visuel. En sciences de l'éducation également, la communication entre le maître et l'élève est envisagée comme un phénomène d'interaction à la fois verbale et non verbale (De Landsheere \& Delchambre, 1979 ; Pujade-Renaud, 1983).

Plus précisément, d'après Corraze (1980), les CNV regroupent "l'ensemble des moyens de communication existant entre des individus n'usant pas du langage verbal ou de ses dérivés non sonores (écrits, langage des sourds muets, etc.) » (p. 12). Les CNV désignent alors un mode de communication, conscient ou non, qui peut prendre une importante diversité de formes, c'est-à-dire qu'elle se manifeste sous différents aspects : gestes, postures, tensions du corps, mouvements, expressions faciales, regards, sourires, proxémie, toucher... (Genevois, 1992). A ce titre, ces formes de CNV ont fait l'objet de nombreuses classifications, dont certaines sont particulièrement englobantes, à l'image de celle de Bonaiuto (2007) qui distingue, au sein des CNV, l'aspect extérieur (par exemple, morphologie), le comportement spatial (par exemple, proxémie), le comportement cinétique (par exemple, gestualité), la face (par exemple, expressions), les signes vocaux (par exemple, silence). Finalement, dans notre étude, la définition des CNV est celle d'un corps communiquant qui inclut cette diversité de formes, en accord avec l'idée que, par le 


\section{eJRIEPS 37 janvier 2016}

corps, nous ne pouvons pas ne pas communiquer (Watzlawick, 1988), c'est à dire produire des signes ayant du sens pour ceux qui les reçoivent.

Ces formes de CNV sont considérées comme indissociables, tout comme les relations entre CNV et CV (Dinica, 2014). A ce titre, Argyle (1972) montre qu'il existe trois relations entre CNV et CV: (1) certaines CNV sont utilisées comme un support de la CV ; (2) d'autres CNV sont utilisées à des fins de remplacement de la CV ; (3) les dernières ont trait aux attitudes, aux émotions et aux manipulations de la situation sociale immédiate. Ramené à l'enseignement, l'ensemble de ces formes de CNV ont aussi été mises en relation avec leurs fonctions en classe. A ce propos, Klinzing et Jackson (1987) ont fait un bilan des travaux ayant étudié les CNV des enseignants, affirmant qu'elles comportent plusieurs fonctions dans l'activité des enseignants, c'est-à-dire plusieurs rôles : (1) communiquer leurs attentes aux élèves ; (2) réguler les interactions en classe ; (3) promouvoir la compréhension des élèves vis-à-vis des contenus.

Enfin, Sperber et Wilson (1989) soulignent qu'une action de communication est pertinente si elle s'inscrit au mieux dans les circonstances environnementales où elle se produit. Cette pertinence est liée aux perceptions que le locuteur fait de son environnement et à la capacité du destinataire d'inférer facilement ce que le locuteur veut dire. Dès lors, pour rendre pertinente une communication, le locuteur doit combiner la production d'un énoncé verbal et d'un comportement non-verbal adapté. En sciences de l'éducation, nous sommes d'avis que cette pertinence nécessite d'étudier les formes et les fonctions des CNV en situation réelle de classe, dans la mesure où cela permet de sortir des approches mentalistes et cognitivistes, en situant l'enseignant dans « son milieu naturel » (Tardif \& Gauthier, 2001). Partant de ce constat, plusieurs recherches ont analysé les CNV des enseignants en prenant appui sur les situations réelles de classe.

Dans une perspective didactique, Moulin (2004) s'est par exemple attaché à décrire les différentes formes de communication dont font usage les enseignants exerçant en primaire. A partir d'une méthodologie basée sur l'étude de données vidéo, il montre que les enseignants utilisent des formes diverses de CNV en classe (éléments gestuels, mimiques, apparence, tenue vestimentaire et éléments sonores). L'auteur conclut en interrogeant les fonctions potentielles de ces formes de CNV, notamment en ce qui concerne l'expression de l'autorité en classe, et prône une approche de la violence scolaire par les relations entre le corps de l'enseignant et le corps de l'élève en classe. Dans la même veine théorique, Forest (2008) s'est attaché à décrire l'usage du corps par des enseignants experts exerçant au primaire. L'auteur cible sa recherche sur la 


\section{eJRIEPS 37 janvier 2016}

« proxémie » (Hall, 1984), c'est-à-dire sur l'ensemble des observations concernant l'usage que l'homme fait de l'espace en tant que produit culturel. Forest (2008) propose de parler de «proche et de lointain » de sorte à envisager la proximité comme un jugement de valeurs sur la distance signifiant pour autrui. A l'aide d'une première analyse "à la sourde », consistant à observer les enregistrements vidéo des cours sans le son, l'auteur met en évidence des caractéristiques peu visibles des situations observées. Une deuxième analyse vise la construction d'une intrigue, de manière à décrire les CNV en lien avec le savoir mis en jeu. Les résultats de cette recherche soulignent les fonctions de la proxémie comme techniques didactiques pour accompagner les apprentissages des élèves. Malgré tout, au sein de ces recherches, les fonctions attribuées aux CNV se fondent exclusivement sur des données extrinsèques sans prendre en compte le point de vue de l'acteur en situation. Une telle méthode pourrait alors être enrichie d'un recueil des significations de l'enseignant, notamment de ses préoccupations, de manière à compléter l'analyse extrinsèque de son activité, en particulier des fonctions des CNV, et d'étudier plus précisément la pertinence de ces CNV en contexte. En outre, ces recherches n'ont pas pour objet de questionner les relations entre CNV et expertise.

A partir d'une démarche psychosociale, Boizumault et Cogérino (2012) allient quant à elles des données extrinsèques et intrinsèques pour analyser les CNV de huit enseignants d'EPS exerçant en collège. Les auteures ont ainsi décrit la diversité des formes de CNV utilisées par les enseignants en classe (gestuelle, postures, silence, déplacements, proxémie et les mimiques). Des entretiens d'autoconfrontation ont été réalisés avec les enseignants à partir d'un montage vidéo juxtaposant des passages choisis par les auteurs à partir de quatre fonctions des CNV définies a priori: (1) déshumanisée (gestes routiniers) ; (2) communicationnelle (interaction organisationnelle rapidement signifiante pour les élèves) ; (3) technique (gestes sportifs) ; (4) relationnelle (interactions pour apaiser et rassurer). Ces recherches permettent selon nous d'affiner les liens entre les formes de CNV produites en classe et les fonctions de ces communications pour les enseignants, à l'aide d'entretiens réalisés avec eux après l'enregistrement des cours. Néanmoins, il nous semble possible de pointer cinq limites à cette étude : (1) la description des formes de CNV ne s'appuie pas sur une analyse complexe mettant en lien ces communications entre elles dans un contexte particulier ; (2) la mise en évidence des fonctions des CNV repose sur des classifications définies a priori par les auteurs et ne découlent pas de l'entretien d'autoconfrontation avec les enseignants ; (3) les relations entre la CNV et la CV de l'enseignant sont peu explicitées, ce qui contribue à scinder 


\section{eJRIEPS 37 janvier 2016}

l'activité de l'enseignant et à isoler les CNV d'autres formes de langage ; (4) les entretiens d'autoconfrontation ne portent pas sur la totalité du cours mais sur une sélection d'extraits vidéo définis a priori par les auteurs, limitant la prise en compte du caractère temporellement situé des CNV au sein de l'histoire de classe ; (5) L'étude de la pertinence des CNV est limitée, dans la mesure où les CNV ne sont pas systématiquement mises en lien avec des contraintes de l'environnement sur l'activité de l'enseignant et des effets sur cet environnement des CNV produites. Pour terminer, cette étude n'a pas eu pour objet de questionner les relations entre CNV et expertise de l'enseignant.

Dans une approche didactique, Kammoun et Amade-Escot (2007) ont cherché à décrire les formes et les fonctions de la gestualité de deux enseignants d'EPS, d'expérience similaire mais d'expertise contrastée. Une focale a été réalisée sur la gestualité de l'enseignant, qui participe et accompagne les processus interactifs en classe. Les auteurs se sont appuyés sur cinq formes de gestes mis en évidence de façon préalable (Mahut, 2003), des « gestes spatio-indicielles » (lorsque l'enseignant par exemple désigne d'un geste de la main l'élève qui doit travailler) aux « gestes symboliques », reconnus par l'ensemble de l'institution classe. Dans un second temps, ils ont proposé un codage à partir du croisement des données d'observation, d'interactions verbales en classe et des transcriptions d'entretiens des enseignants, afin d'affecter à chaque catégorie gestuelle une ou plusieurs fonctions dans le processus didactique, de « définir» (pour que les élèves sachent à quel jeu ils vont jouer), à « institutionnaliser » (pour que la connaissance soit légitime, vraie et reconnue dans l'institution classe). Cette recherche permet de comprendre le rôle de l'expertise dans la manière d'utiliser le corps en classe. Pour l'enseignant polyvalent, la gestualité contribue essentiellement à l'organisation spatiale et à la répartition des élèves dans le gymnase, alors que pour l'expert, elle porte également sur des variables précises de la situation d'apprentissage. Toutefois, la notion d'expertise renvoie dans cette étude prioritairement à la connaissance de l'APSA enseignée, ce qui peut être limitant au regard de sa complexité et de son caractère fondamentalement située. En effet, Visioli et Ria (2010) considèrent que la spécialisation dans l'APSA ne constitue, au même titre que l'expérience, qu'une composante de l'expertise. Cette dernière englobe en fait à la fois l'expérience, la connaissance didactique, la connaissance pédagogique, la pratique réflexive et la reconnaissance sociale (Tochon, 1993).

Dans une perspective didactique, Mahut, Mahut, Gréhaigne et Masselot (2005) se sont intéressés à la gestuelle de l'enseignant en natation et à la co-construction de sens avec 


\section{eJRIEPS 37 janvier 2016}

des étudiants. Cette étude s'ancre dans le constat d'une très forte utilisation des gestes illustratifs dans la communication dans l'interaction enseignant / élève en EPS par rapport à une conversation normale. Elle consiste à comparer le sens accordé à des gestes iconiques spécifiques de la natation par l'enseignant expérimenté et spécialiste de l'APSA, et des étudiants ayant suivi son enseignement ou non. Les résultats soulignent l'idée que si la production gestuelle constitue pour l'enseignant d'EPS un outil essentiel pour illustrer et orienter son intention didactique, il existe un fort décalage des interprétations de signes entre producteur et interprétant du langage véhiculant les contenus d'enseignement. Cette recherche ouvre donc à la question de l'effet des gestes de l'enseignant sur les élèves, dans le prolongement de la théorie des actes de discours (Austin, 1970), qui envisage l'acte perlocutoire comme l'obtention de certains effets par la parole ou par les comportements non-verbaux. La question de la pertinence des CNV constitue, à ce titre, une préoccupation majeure au sein de cette recherche. Néanmoins, en ciblant les gestes iconiques, elle n'envisage pas les différentes formes de CNV dans leur globalité. D'autre part, la focale sur les enjeux d'apprentissages spécifiques à la natation ne permet pas d'envisager d'autres fonctions des CNV en classe, notamment liées à la gestion de classe et aux apprentissages en termes d'attitude et de méthode.

Les travaux menés dans une perspective située attachent de l'importance à la prise en compte du contexte dans l'activité des acteurs en classe, sans la scinder à partir de classifications définies a priori (par exemple, « gestion de classe » vs « apprentissage »). Gal-Petitfaux (2011) a tenté de rendre compte de l'activité de CV et de CNV d'enseignants d'EPS définis comme « chevronnés », face à des élèves qui nagent en «file indienne » dans des lignes d'eau situées en bordure de bassin. Les données recueillies permettent la description de l'organisation comportementale des enseignants, c'est-à-dire des patterns (déplacements, postures, forme et contenus de communication) qu'il produit pour interagir avec les élèves, en lien avec une description de la signification de ces comportements. II ressort que les enseignants parviennent à canaliser l'attention du groupe en adaptant leur placement par rapport aux élèves et que, par leurs comportements, ils rendent explicites pour les élèves leurs intentions de les surveiller et de communiquer avec eux. Cette étude, notamment en mettant en lien le point de vue intrinsèque de l'enseignant chevronné et des observations extrinsèques réalisées par la chercheuse, participe d'une « analyse du corps entier » (Burel \& Andrieu, 2014). Elle nous invite alors à préciser l'observatoire des CNV pour approfondir l'analyse de leurs formes, de leurs fonctions, et de leur pertinence en lien avec le contexte de classe. 


\section{eJRIEPS 37 janvier 2016}

Finalement, l'examen de la littérature portant sur l'analyse des CNV de l'enseignant en situation réelle, étudiées dans des perspectives théoriques diverses, nous invite à envisager plusieurs axes d'approfondissements. D'un point de vue intrinsèque, il s'agirait d'enrichir : (1) l'étude des préoccupations de l'enseignant, ce qui permettrait de préciser les formes et les fonctions des CNV en situation ; (2) l'étude de la pertinence des CNV à travers les perceptions des enseignants en situation de classe, ce qui permettrait de comprendre ce qui les amène à produire des CNV particulière et quels effets ils perçoivent de leurs CNV sur les élèves. L'étude de leurs connaissances mobilisées en situation peut aussi nous permettre d'analyser la pertinence des CNV en lien avec son expertise. D'un point de vue extrinsèque, il s'agirait d'enrichir : (1) l'étude des formes de CNV comme un tout intégré entre elles et avec la CV ; (2) les relations entre les formes et les fonctions des CNV de l'enseignant et la temporalité des leçons ; (3) les relations entre les formes et les fonctions des CNV de l'enseignant et les configurations d'activité collective en classe ; (4) l'évaluation de la pertinence des CNV au regard des comportements des élèves.

D'un point de vue empirique, l'objectif de cette recherche est : (1) d'analyser les formes et les fonctions des CNV produites par des enseignants d'EPS experts en situation de classe: (2) de mettre en lien les formes et les fonctions des CNV avec les CV des enseignants en situation; (3) de mettre en lien les formes et les fonctions des CNV de l'enseignant avec ses perceptions et ses connaissances mobilisées en situation, de manière à discuter de la pertinence des CNV en classe.

\section{Cadrage théorique}

Nous avons fait le choix d'une approche "entrée activité ", considérée comme un outil intéressant pour l'analyse des communications quotidiennes et professionnelles (Barbier \& Durand, 2006). Dans cette perspective, l'activité humaine est entendue dans la diversité de ses conditions d'exercice, dans l'historicité, la singularité et l'inédit de sa survenance, et dans l'unité que lui donne le fait qu'elle est développée par des sujets humains (Barbier \& Durand, 2003). Le dilemme est alors de conserver autant que possible le caractère complexe, pluridimensionnel, de totalité signifiante de la pratique, tout en la réduisant afin de la rendre accessible par les démarches, méthodes et concepts scientifiques (Durand, 2008). Dans notre étude, il s'agit alors d'envisager la définition des CNV de manière suffisamment globale et complexe tout en ciblant les critères d'analyse des CNV des enseignants engagés dans l'activité réelle. Plus précisément, nous faisons le choix du cadre théorique du « cours d'action » (Theureau, 2006), pour quatre raisons : 


\section{eJRIEPS 37 janvier 2016}

(1) ce cadre théorique s'appuie sur l'idée que l'activité humaine est incarnée. En ce sens, « toute séparation entre corps et esprit - entre perception, émotion et action ressortant du corps et cognition ressortant de l'esprit - est considérée comme non pertinente» (Theureau \& Donin, 2005, p. 2). Le cadre du cours d'action s'inscrit de ce fait dans la lignée des chercheurs soulignant l'importance de l'inscription corporelle de l'esprit (Varela, Rosch \& Thompson, 1993). L'idée centrale est que l'esprit se trouve dans la codétermination de l'intérieur et de l'extérieur du corps, et ne peut en aucun cas être considéré comme séparé de lui.

(2) ce cadre théorique prône une conception située de l'activité d'un acteur, c'est à dire qu'elle est indissociable du contexte (Suchman, 1987). Le couplage structurel entre l'individu et la situation se transforme en permanence au cours de l'activité, qui émerge d'un effort d'adaptation à un contexte dont les éléments significatifs pour l'acteur constituent des ressources qu'il utilise pour agir (Varela, 1989). Egalement, le courant de recherche développé sous l'appellation d'activité située est « une entrée essentielle pour comprendre l'expertise dans un domaine particulier, et donc la manière dont la compétence s'exprime dans les environnements complexes » (Delignières, 2009, p. 50).

(3) ce cadre théorique accorde une importance majeure à la singularité de l'action. Entrer par une « science du singulier » constitue une forme de questionnement ouvert qui essaie de ne pas investiguer la réalité étudiée à partir de catégories préétablies et de laisser la possibilité aux évènements de se manifester dans leur propre nature (Vermersch, 2000). Pour l'auteur, le « une fois » a autant de puissance de détermination que le million d'occurrence. Autrement dit, ce n'est pas la multiplicité qui a le pouvoir en soi, le « un », à travers des approches de type études de cas, en a tout autant.

(4) ce cadre théorique articule des données intrinsèques et extrinsèques. En accordant le primat à l'intrinsèque, Theureau (2006) considère le « cours d'expérience », c'est à dire la construction de sens pour l'acteur tout au long de son activité, comme un préalable nécessaire avant l'investigation d'autres objets théoriques. Cette idée s'appuie sur le présupposé qu'il existe un couplage asymétrique entre l'individu et l'environnement, de sorte que l'activité est orientée par la perspective de l'acteur (Maturana \& Varela, 1994). Le « cours d'action » inclut alors le cours d'expérience auquel sont ajoutés les contraintes qui pèsent sur l'activité et les effets extrinsèques qui en découlent (Theureau, 2006).

D'un point de vue théorique, l'objectif est d'analyser les formes, les fonctions et la pertinence des CNV d'enseignants experts à partir: (1) de l'étude de leur cours d'expérience, de manière à analyser leurs CNV à partir de leur point de vue intrinsèque ; 


\section{eJRIEPS 37 janvier 2016}

(2) de l'étude de leur cours d'action, de manière à ancrer leurs CNV dans la complexité du contexte de classe et des contraintes/effets extrinsèques en relation à leur activité.

\section{Méthodologie}

3. 1. Participants et situations étudiées

Cinq enseignants « experts » en EPS, âgés de 36 à 60 ans et d'une expérience dans l'enseignement de 10 à 39 ans, se sont portés volontaires pour participer à l'étude. De manière à sélectionner ces participants, nous nous sommes appuyés sur les propositions de Tochon (1993), reprises par Visioli et Ria (2010), qui établit cinq critères d'expertise : (1) l'expérience professionnelle ; (2) la connaissance didactique ; (3) la connaissance pédagogique ; (4) la pratique réflexive ; (5) la reconnaissance sociale. Chaque enseignant a été observé lors de deux cours d'EPS, avec des classes de collège et de lycée. Chaque enseignant a été invité à choisir deux APSA: (1) l'une dans laquelle il se sentait spécialiste ; (2) l'autre dans laquelle il se sentait non spécialiste. Seul un enseignant a été filmé dans un seul cours d'EPS. Ce choix s'explique par notre volonté de ne pas négliger l'influence qu'une spécialisation plus ou moins importante dans l'APSA enseignée pourrait exercer sur les CNV en classe. Toutefois, le statut accordé à la spécialisation dans l'APSA est secondaire, cette spécialisation restant une des composantes de l'expertise. Les conditions de la collaboration ont été précisées dès le départ de la recherche afin d'instaurer une relation de confiance entre le chercheur et les participants.

\section{2. Recueil des données}

Deux types de données ont été recueillies : (1) des données d'observation et de verbalisation in situ ; (2) des données de verbalisation a posteriori de l'action ; (3) des données d'entretien bilan à la fin de la recherche.

\section{2. 1. Des données d'observation et de verbalisation in situ}

Ces données ont trait à l'enregistrement des enseignants et des élèves en situation de classe. Elles ont été obtenues à l'aide d'une caméra numérique sur pied et d'un micro HF porté par l'enseignant, et recueillies en continu, de l'arrivée dans le gymnase au départ des élèves aux vestiaires, afin de restituer le caractère dynamique du cours d'action et en particulier des CNV en classe.

\section{2. 2. Des données de verbalisation a posteriori de l'action}

$\mathrm{Au}$ cours de ces entretiens, chaque enseignant a été invité à expliciter, montrer et commenter les éléments significatifs pour lui lors du cours d'action (Theureau, 2006). Ces entretiens ont consisté pour le chercheur à questionner l'enseignant sur ses 


\section{eJRIEPS 37 janvier 2016}

préoccupations, mais aussi sur ses perceptions et ses connaissances durant le cours. Les CNV n'ont pas fait l'objet d'une définition a priori pour les enseignants ni d'un questionnement spécifique du chercheur, qui auraient pu influencer la nature des données recueillies. Les enseignants se sont donc prononcés spontanément sur les CNV qui étaient significatives pour eux durant le cours. Chaque entretien a été réalisé au maximum trois jours après le recueil des données d'observation in situ.

3.2.3. Des données d'entretien bilan à la fin de la recherche

A la fin de la recherche, nous avons réalisé un entretien bilan avec chaque enseignant, de manière à recueillir ses impressions a posteriori et à renseigner d'éventuels rôles transformatifs joués par leur participation à cette recherche. L'entretien s'est déroulé sous une forme semi-directive consistant à les questionner de manière large et ouverte sur ce qu'il retenait de cette participation. Ces données n'ont pas pour vocation d'être traitées de manière spécifique, mais visent à mettre en perspective l'importance d'étudier le caractère souvent inconscient des CNV dans de futures recherches.

\section{3. Traitement des données}

Deux types de traitement de données ont été effectués, lors de moments saillants du cours d'action des enseignants, entendus comme des "évènements remarquables " (Leutenegger, 2000) du point de vue des CNV produites par l'enseignant. En accord avec le présupposé ontologique consistant à accorder le primat au point de vue des acteurs (Theureau, 2006), ces moments ont été déterminés de façon entièrement inductive, à partir des données de verbalisation des enseignants lors des entretiens d'autoconfrontation. En effet, les CNV ne sont pas présentes en permanence dans le discours des enseignants, qui s'attachent, dans l'entretien, à évoquer les éléments significatifs pour eux dans la situation. Les moments sélectionnés correspondent donc à l'émergence des CNV dans l'explicitation, par les enseignants, de leur activité. Plus précisément, nous avons sélectionné les moments au cours desquels les enseignants ont : (1) insisté sur l'importance de leurs CNV en situation (critère quantitatif) ; (2) ont été précis dans la verbalisation des formes et des fonctions de leurs CNV en situation (critère qualitatif). Ces moments saillants, un par enseignant, ont fait l'objet d'un triple traitement de données : (1) un traitement des données d'observation et de verbalisation in situ : mise en évidence des formes de CNV produites par l'enseignant ; (2) un traitement des données de verbalisation après l'action: mise en évidence des fonctions des CNV produites par l'enseignant. Les données d'entretien bilan à la fin de la recherche n'ont pas fait l'objet d'un traitement spécifique et seront exposées à titre informatif ; (3) une mise en 


\section{eJRIEPS 37 janvier 2016}

relation des données de verbalisation après l'action et d'observation par le chercheur : la pertinence des CNV produites par l'enseignant

3. 3. 1. Traitement des données d'observation et de verbalisation in situ : mise en évidence des formes de CNV produites par l'enseignant

Le traitement des données d'observation in situ a été réalisé en deux étapes :

(1) un descriptif détaillé des CNV et des CV tout au long du moment saillant. Les formes ont été décrites de manière exhaustive et inductive, en accord avec notre choix d'une définition globale des CNV. Ces CNV ont été décrites à partir des données d'observation et d'un découpage de chaque moment saillant en plans successifs, permettant d'accéder à une description fine des CNV tout au long de ce moment. Suite à cela, les formes des CNV ont été explicitées à l'écrit de manière à pouvoir être repositionnées dans l'histoire du moment saillant. Chaque forme de CNV a été mise en gras pour plus de visibilité ;

(2) la schématisation de la configuration de l'activité collective en classe permettant une description fine du contexte humain et matériel à chaque moment saillant ;

3. 3. 2. Traitement des données de verbalisation après l'action : mise en évidence des fonctions des CNV produites par l'enseignant

Le traitement des données qualitatives de verbalisation après l'action a consisté à reconstruire le cours d'expérience de chaque enseignant au moment saillant. Cette reconstruction a été réalisée en quatre étapes :

(1) la constitution d'un protocole à deux volets mettant en correspondance les données d'observation et les données de verbalisation retranscrites ;

(2) la mise en forme du récit réduit (Theureau, 1992) du cours d'expérience des enseignants en présentant l'enchaînement des unités significatives élémentaires (par exemple, « regroupement des élèves par l'enseignant ») ;

(3) la documentation du signe hexadique (Theureau, 2006), qui décrit la conscience préréflexive d'un acteur donné à un instant donné, sur la base de six composantes, chacune décrivant une catégorie de l'expérience de l'acteur à chaque instant de son activité : (a) l'unité significative élémentaire $(U)$ du cours d'expérience est la fraction de l'activité qui est montrée, racontée et commentée par l'acteur ; (b) l'engagement (E) dans la situation a trait aux préoccupations de l'enseignant dans la situation ; (c) le représentamen (R) est ce qui fait signe pour l'acteur dans la situation ; (d) l'actualité potentielle $(A)$ regroupe ce qui est attendu par l'acteur dans la situation, compte tenu de l'engagement ; (e) le référentiel (S) a trait aux éléments de connaissance issus des cours d'expérience passés et 


\section{eJRIEPS 37 janvier 2016}

mobilisés par l'acteur à un instant $\mathrm{t}$; (f) l'interprétant (I) est la construction d'éléments de connaissance (validation ou invalidation) de l'acteur dans la situation.

(4) Après avoir documenté ces six catégories, nous nous sommes focalisés sur les préoccupations de l'enseignement dans la situation, de manière à renseigner les fonctions des CNV en classe, du point de vue de l'enseignant. Autrement dit, dans cette recherche, les fonctions des CNV sont entendues du point de vue de l'enseignant, à partir de ses préoccupations en situation. Elles ont été mises en gras dans le texte pour plus de visibilité ;

3. 3. 3. Mise en relation des données de verbalisation après l'action et d'observation par le chercheur: la pertinence des CNV produites par l'enseignant

La pertinence des CNV en situation a été décrite à partir d'une mise en relation des données de verbalisation après l'action et d'observation par le chercheur. Trois étapes ont guidé cette description :

(1) en ce qui concerne les données de verbalisation a posteriori, nous nous sommes, d'une part, focalisés sur le représentamen $R$ qui renseigne les causes et les effets des CNV en classe, du point de vue de l'enseignant. D'autre part, nous nous sommes centrés sur le Référentiel $S$ qui traduit les connaissances, mobilisables en lien notamment avec les CNV de l'enseignant en classe. Autrement dit, le représentamen et le référentiel nous permettent de renseigner la pertinence des CNV en situation du point de vue de l'enseignant ;

(2) en ce qui concerne les données d'observation, nous avons inscrit les CNV dans la dynamique temporelle du cours en décrivant les contraintes et les effets extrinsèques pesant sur l'activité de l'enseignant lors des moments saillants. Les contraintes renvoient aux éléments ayant générés la production de CNV (par exemple, les comportements inattentifs d'un élève) tandis que les effets renvoient aux conséquences des CNV sur la situation et particulièrement sur l'activité des élèves (par exemple, le retour au calme de cet élève) ;

(3) la mise en relation des données de verbalisation a posteriori (représentamen et référentiel) et des données d'observation (contraintes et effets extrinsèques) de manière à comparer le point de vue de l'enseignant aux évènements réels de classe entourant la production des CNV. 


\section{eJRIEPS 37 janvier 2016}

\section{Résultats}

Les données obtenues au cours de cette étude sont structurées en cinq parties, renvoyant chacune à une étude de cas décrivant l'activité des cinq enseignants à un moment du cours durant lequel il a utilisé les CNV face aux élèves : (1) Etude de cas $n^{\circ} 1$ : formes et fonctions des CNV de Paul en cours de danse (non spécialiste) avec une classe de cinquième ; (2) Etude de cas $\mathrm{n}^{\circ} 2$ : formes et fonctions des CNV de Catherine en cours de danse (non spécialiste) avec une classe de première ; (3) Etude de cas $n^{\circ} 3$ : formes et fonctions des CNV de François en cours de arts du cirque (non spécialiste) avec une classe de quatrième ; (4) Etude de cas $n^{\circ} 4$ : formes et fonctions des CNV de Christophe en cours de tennis de table (spécialiste) avec une classe de première ; (5) Etude de cas n`5 : formes et fonctions des CNV de Philippe en cours de danse (non spécialiste) avec une classe de sixième.

4. 1. Etude de cas $n^{\circ} 1$ : formes et fonctions des CNV de Paul en cours de danse (non spécialiste) avec une classe de cinquième

L'étude de cas $\mathrm{n}^{\circ} 1$ propose une analyse de l'activité de Paul lors du quatrième cours de danse d'un cycle de dix leçons, avec 23 élèves de cinquième d'un collège situé en zone d'éducation prioritaire. Lorsque Paul a débuté le cours, il venait d'être contraint à changer au dernier moment d'installation sportive, se retrouvant sous pression temporelle au début de la leçon dans une salle qu'il ne connaissait pas. D'emblée, il a regroupé les élèves et insisté sur la nécessité de se montrer efficace dans le travail et l'organisation, en raison du temps perdu en début de séance. II s'est ensuite attaché à présenter l'objectif de la leçon : dans un premier temps, il s'agit de permettre aux élèves de préparer par groupe de deux ou trois une chorégraphie synchronisée, composée de trois phrases de dix temps chacune; dans un second temps, il s'agit pour les élèves de réaliser la chorégraphie devant la classe, et d'évaluer les prestations des autres groupes. L'étude de cas $n^{\circ} 1$ se situe entre le moment où Paul interpelle un élève qu'il perçoit comme non attentif lors des explications, et le moment où il reprend ses explications liées au contenu de la leçon. Ce moment, situé au début du cours, a lieu lors de la dixième minute (10'10" à 11'10') (Figure $1)$. 


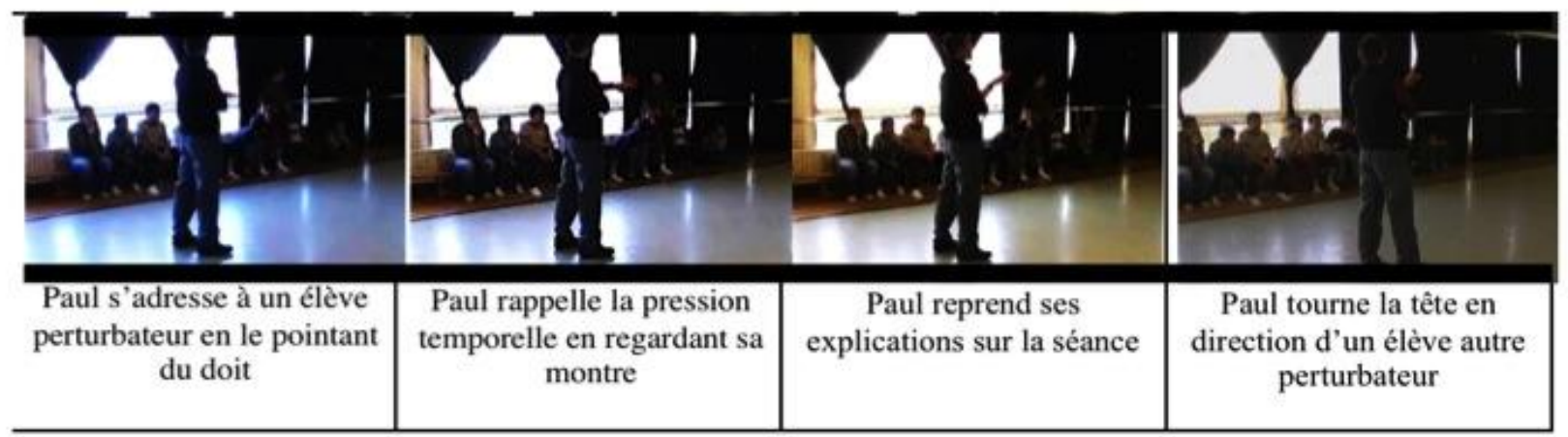

Figure 1. Description de l'activité de Paul (étude de cas $n^{\circ} 1$ ).

Lors de cette configuration d'activité collective, l'enseignant et les élèves sont positionnés en regroupement : l'enseignant est debout et fait face aux élèves. Les garçons sont assis sur un banc à sa gauche, alors que les filles sont placées sur un banc sur sa droite (Figure 2).

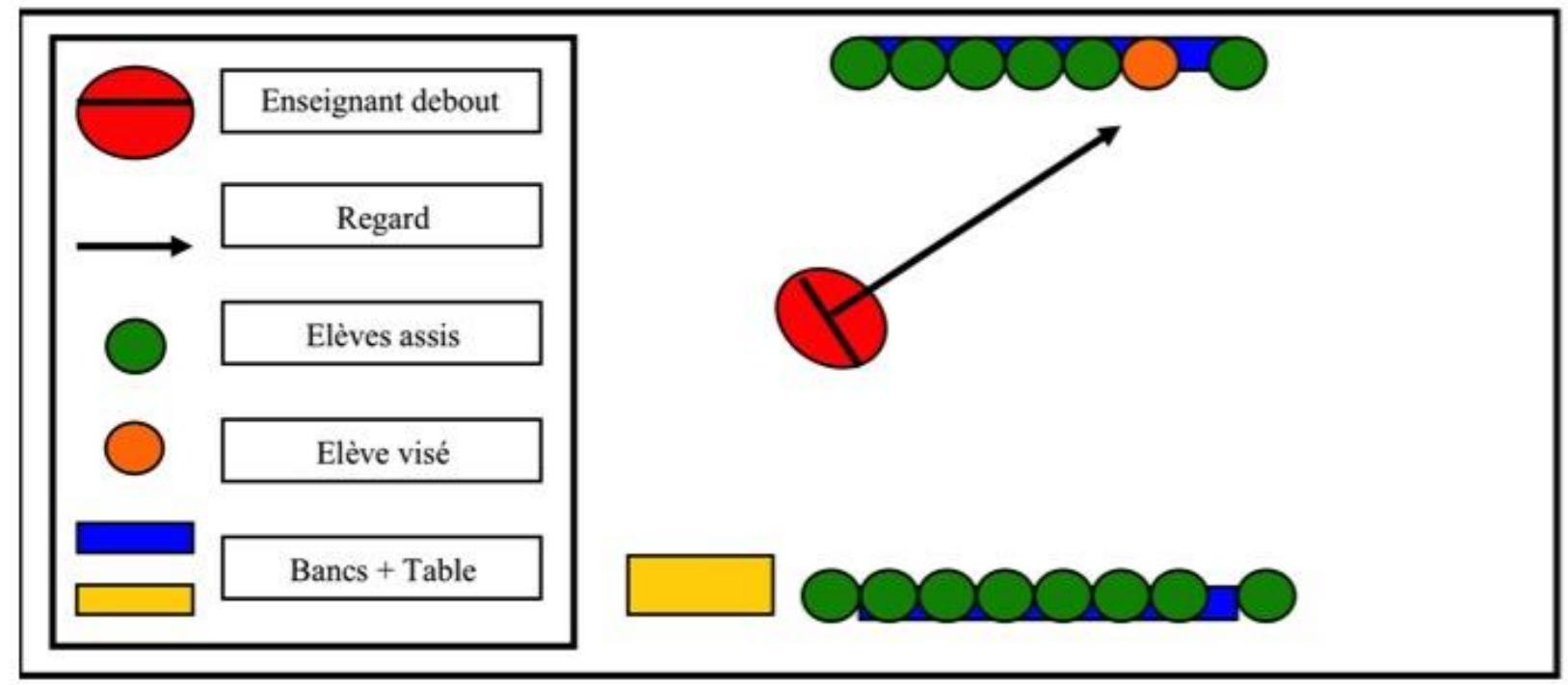

Figure 2. Schéma de la configuration de l'activité collective (étude de cas $\mathrm{n}^{\circ} 1$ ).

4. 1. 1. Description des données d'observation et de verbalisation in situ : les formes de CNV

Dans un premier temps, Paul cible un élève et lui demande de se calmer : "je t'ai déjà prévenu tout à l'heure: tu te calmes! ». L'enseignant complète cette CV par plusieurs formes de CNV : il montre l'élève du doigt, se tient très droit le corps orienté face à lui, le fixe du regard et termine son propos en marquant un cours temps de silence. Dans un deuxième temps, Paul s'adresse à tous les élèves et dit : «bien, je vous ai déjà dit, 10h57, donc le temps tout à l'heure de se changer et rentrer au collège autant dire que l'on a à peine 50 minutes de cours. Donc on va essayer d'être efficace ". En disant cela, l'enseignant regarde ostensiblement sa montre. Dans un troisième temps, Paul reprend le fil de son explication liée au déroulement de la leçon de danse, en accompagnant ses 


\section{eJRIEPS 37 janvier 2016}

paroles de gestes de la main. Enfin, dans un dernier temps, Paul tourne la tête en direction d'un autre élève qui se montre inattentif à ses explications en discutant avec un camarade. II interrompt son explication, le fixe du regard en marquant un silence, sans communiquer verbalement avec lui (Figure 1).

4. 1. 2. Description des données de verbalisation a posteriori : les fonctions des CNV

Les données obtenues lors de l'entretien d'autoconfrontation montrent que lors du premier temps, les préoccupations de l'enseignant sont de plusieurs ordres, illustrant les fonctions données aux CNV en situation. II souhaite tout d'abord cadrer l'élève qu'il perçoit comme inattentif, mais il veut également prendre un élève comme exemple pour recadrer l'ensemble de la classe : «tu vois j'ai fait une intervention sur un élève, moi je fais souvent ça tout de suite pour cadrer (...) je sentais qu'il fallait cadrer à mort sur ce début de séance (...) il y a une volonté de ma part, vu le climat antérieur, sur le trajet, sur la cour, surtout les gars là, de cadrer ». Dans un deuxième temps, l'enseignant cherche à rappeler aux élèves que du temps a été perdu en début de leçon et qu'il est nécessaire d'être attentif aux consignes pour ne pas en perdre plus dans le reste du cours. Dans un troisième temps, l'enseignant reprend rapidement les explications quant au contenu de la leçon, en cherchant à être le plus clair possible : «mon intention, c'est de clarifier, de dire clairement ce que j'attends, surtout sur la première situation, enfin j'explique d'ailleurs les deux temps de séance, donc toujours à présenter mes intentions avec des mots qu'ils comprennent, ce n'est pas évident ce décalage, qu'ils sachent clairement ce qu'on va faire aujourd'hui ». Plus généralement, lors de ce dernier temps, Paul exprime la préoccupation de montrer aux élèves la nécessité de conserver le silence lorsqu'il parle, au même titre qu'il les écoute lorsqu'ils ont quelque chose à dire : «ben c'est à dire que je veux arriver à cet état où je peux prendre la parole dans un climat où je pense que les élèves peuvent m'écouter. C'est à dire pour moi c'est le silence, avec des gamins qui sont quand même attentifs, ils ont un regard vers moi. Au moins il y a ça, moi je ne peux pas... je trouve que c'est un manque de respect, et je leur dis souvent, moi quand tu me parles, je t'écoute, je ne suis pas en train de faire autre chose... Cette égalité relationnelle, j'insiste toujours làdessus ». Dans un dernier temps, Paul recadre à nouveau un élève inattentif, en utilisant cette fois-ci uniquement le regard. Ces préoccupations sont alors semblables à celles exprimées précédemment, en lien avec la volonté de cadrer l'élève et de présenter la leçon sans perdre de temps (Tableau 1).

Tableau I : Récit réduit du cours d'action de Paul (étude de cas n¹) 
eJRIEPS 37 janvier 2016

\begin{tabular}{|c|c|c|}
\hline Temps & Elémentaires & Préoccupations (eR) \\
\hline $10 " 10$ & $\begin{array}{l}\text { Paul s'adresse à un élève } \\
\text { perturbateur en le pointant du } \\
\text { doit }\end{array}$ & $\begin{array}{l}\text { - Faire en sorte que l'élève se calme } \\
\text { - Cadrer l'ensemble des élèves en début de leçon } \\
\text { - Présenter clairement la leçon pour ne pas perdre de } \\
\text { temps }\end{array}$ \\
\hline $10 " 15$ & $\begin{array}{l}\text { Paul rappelle la pression } \\
\text { temporelle en regardant sa } \\
\text { montre }\end{array}$ & $\begin{array}{l}\text { - Rappeler aux élèves la nécessité de ne pas perdre de } \\
\text { temps et d'être efficace }\end{array}$ \\
\hline $10 " 18$ & $\begin{array}{l}\text { Paul reprend ses explications } \\
\text { sur la séance }\end{array}$ & $\begin{array}{l}\text { - Présenter clairement la leçon pour ne pas perdre de } \\
\text { temps } \\
\text { - Instaurer un respect quant à l'écoute de la personne } \\
\text { qui parle }\end{array}$ \\
\hline $11 " 03$ & $\begin{array}{l}\text { Paul s'adresse à un élève } \\
\text { perturbateur en le fixant du } \\
\text { regard }\end{array}$ & $\begin{array}{l}\text { - Faire en sorte que l'élève se reconcentre } \\
\text { - Cadrer l'ensemble des élèves en début de leçon } \\
\text { - Présenter clairement la leçon pour ne pas perdre de } \\
\text { temps }\end{array}$ \\
\hline
\end{tabular}

4. 1. 3. Relations entre les CNV et le contexte de classe : la pertinence des CNV

D'après le point de vue de Paul, les CNV ont été produites consécutivement à un certain nombre de perceptions de la situation de classe. Son représentamen est : (1) l'agitation d'un élève en particulier mais également de la classe en général ; (2) les gestes et les regards des élèves («je suis attentif aux gestes des élèves, aux regards"). Les CNV ont également émergé à partir des connaissances mobilisées par Paul en situation de classe. Son référentiel a trait : (1) à la nécessité de prévenir l'inattention des élèves en classe (« je vois ce qui se passe. Et ça c'est super important, surtout quand tu as des gamins qui, dès qu'ils bougent un peu... ») ; (2) à la fermeté vis-à-vis des comportements inattentifs en classe (« moi je sais, dans mon discours je sais ce que je veux. J'arrive avec l'expérience à pouvoir dire ce que j'ai à dire »).

Les perceptions de Paul lui permettent également de mesurer les effets positifs de ses CNV produites. Par exemple, lorsqu'il tourne la tête vers l'élève inattentif, son représentamen est : " je viens de voir que sa tête commence à tourner, je tourne la tête. Mais rien que le fait de tourner la tête, il a commencé à revenir vers moi. C'est ça qu'il faut tout le temps gérer, entre ton discours que tu dois maîtriser et clarifier, et en même temps ce regard ".

D'après l'analyse extrinsèque de la situation de classe entourant l'étude de cas, nous remarquons que certains élèves, bien qu'assis conformément à l'organisation spatiale souhaitée par l'enseignant (élèves assis sur les bancs de part et d'autre de lui), témoignaient de comportements inattentifs (regards non portés sur l'enseignant, 


\section{eJRIEPS 37 janvier 2016}

bavardages discrets, sourires, etc.). Suite à l'intervention de l'enseignant sur un de ces élèves, les comportements inattentifs ont directement cessé.

Finalement, nous constatons que les perceptions de l'enseignant se vérifient par l'analyse extrinsèque de la situation de classe.

\section{1. 4. Analyse des données de l'entretien bilan}

L'entretien bilan réalisé avec Paul a permis de mettre en évidence une certaine surprise de l'enseignant concernant son engagement en classe, et particulièrement au sujet de ce que son corps donne à voir aux élèves : «moi, l'idée que je ressors de ton intervention, c'est de me voir, et de me voir, je me surprends: j'ai l'impression que c'est moi, je me reconnais, mais j'ai l'impression d'être dans un engagement perpétuel, et je me dis que la progression peut-être dans cette intelligence émotionnelle, ça serait d'aller encore vers une plus grande prise de distance. J'ai l'impression d'être tout le temps à l'affût, de ne rien laisser passer; alors c'est peut-être le contexte, l'aspect situé, mais moi ce que j'en retiens quand j'ai vu ça, c'est il faut que je me calme quoi... II faut que je prenne plus de distance, je vais pas tenir trente ans comme ça... ».

4. 2. Etude de cas $n^{\circ} 2$ : formes et fonctions des CNV de Catherine en cours de danse (non spécialiste) avec une classe de première

L'étude de cas $\mathrm{n}^{\circ} 2$ propose une analyse de l'activité de Catherine lors du troisième cours de danse d'un cycle de douze leçons, avec une classe de première composée de 26 élèves dont 24 filles. Catherine a débuté le cours par un bref regroupement au cours duquel elle est revenue sur des observations réalisées par les élèves lors de la leçon précédente. Elle a aussi présenté aux élèves l'objectif de la séance, consistant à enrichir des esquisses de chorégraphies par groupes de 4/5 élèves, en travaillant à stabiliser les équilibres, diversifier les déplacements, diversifier la gestuelle et exploiter l'espace scénique. Simultanément, il s'agit pour les élèves de s'organiser collectivement dans le cadre d'un processus de création aboutissant à la fin de la séance à la présentation d'une œuvre commune face à la classe. Après avoir mis en relation le projet de leçon dans le prolongement de ce qui a été réalisé la semaine précédente, et plus globalement dans la perspective de réalisation d'une chorégraphie en fin de cycle, Catherine a expliqué les consignes de la situation d'échauffement. II s'agit pour les élèves de réaliser des déplacements aléatoires dans la salle, sur fond sonore, puis de s'immobiliser à l'arrêt de la musique. Les élèves se sont ensuite mis en activité et Catherine, placée à proximité du meuble Hifi, a commencé par rythmer cette activité en lançant et en stoppant la musique. L'étude de cas $n^{\circ} 2$ se situe entre le moment où Catherine s'éloigne du meuble Hifi pour 


\section{eJRIEPS 37 janvier 2016}

aller se déplacer au milieu de la salle, et qu'elle produit un certain nombre de formes de CNV, consistant principalement à utiliser la proxémie, au début du cours, lors de l'échauffement (de 3'50" à 4'16").

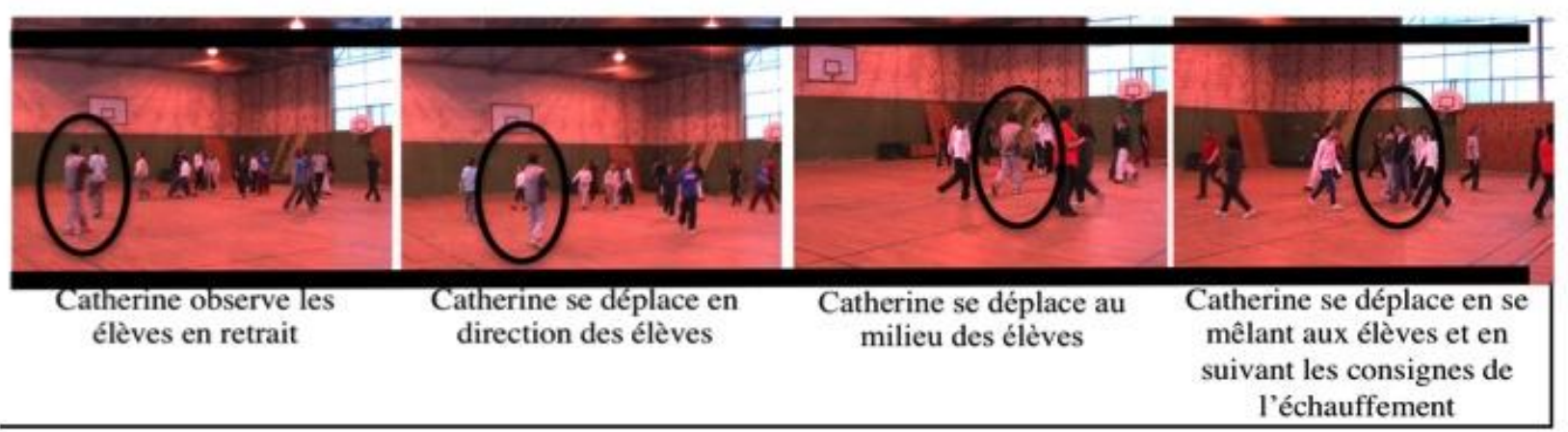

Figure 3. Description de l'activité de Catherine (étude de cas $\mathrm{n}^{\circ} 2$ ).

Dans cette configuration d'activité collective, les élèves sont dispersés dans la salle, marchant sur la musique et s'immobilisant à son arrêt. L'enseignante démarre quant à elle en retrait et se mêle progressivement à eux (Figure 4).

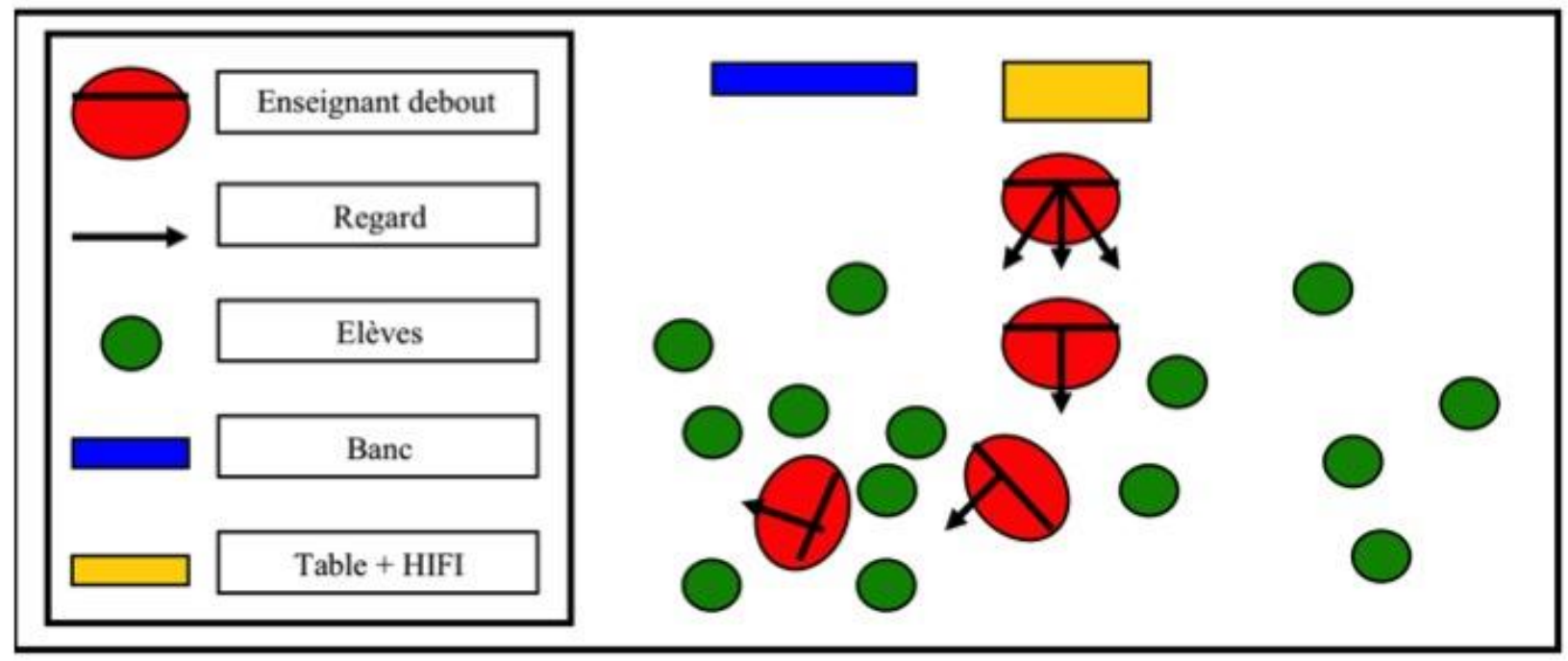

Figure 4 : Schéma de la configuration de l'activité collective (étude de cas $n^{\circ} 2$ )

4. 2. 1. Analyse des données d'observation et de verbalisation in situ : les formes de CNV

Dans un premier temps, Catherine reste en retrait par rapport aux élèves et les observe réaliser l'exercice, debout et immobile, la posture droite et le corps orienté vers les élèves, les bras croisés et le regard balayant la salle et l'ensemble des élèves. Dans un deuxième temps, elle se déplace lentement en direction des élèves, les bras toujours croisés et le regard balayant la salle. Dans un troisième temps, elle se situe au milieu des élèves et marche à leur côté. Ses bras sont désormais décroisés et ses mains rejointes dans le dos 


\section{eJRIEPS 37 janvier 2016}

alors que sa tête est tournée légèrement sur sa gauche en direction de certains élèves. Enfin, dans un quatrième temps, Catherine prend part à l'échauffement : elle se déplace de façon aléatoire, se mêlant aux élèves, les mains toujours rejointes dans le dos et le regard fixe devant elle (Figure 3).

4. 2. 2. Analyse des données de verbalisation a posteriori : les fonctions des CNV

L'entretien d'autoconfrontation révèle que dans un premier temps, la préoccupation de Catherine est en premier lieu d'observer les élèves et de sonder leur niveau d'engagement dans l'activité. Elle a senti lors du regroupement que les élèves étaient inquiets de présenter leur chorégraphie au reste de la classe à la fin de la leçon et souhaite s'assurer qu'ils ne fuient pas l'activité danse. Lors les temps suivants, la préoccupation de Catherine est essentiellement de rompre l'aspect frontal de la relation avec les élèves en allant se déplacer au milieu du groupe. Ses CNV, consistant à s'impliquer corporellement en se rapprochant d'eux, ont pour fonction d'influer sur l'ambiance de classe : « Je vais exprès au milieu d'elles. Je veux rompre le frontal, parce que je me dis que ça peut en aider quelques unes qui ne sont pas très à l'aise ". Plus précisément, Catherine souhaite induire un climat de confiance très important lors des cycles Danse: « Je veux leur montrer que l'on travaille ensemble. On ne fait pas que les faire travailler. On travaille avec eux » (Tableau 3).

Tableau II. Récit réduit du cours d'action de Catherine (étude de cas n²).

\begin{tabular}{lll}
\hline Temps & Unités Elémentaires (UE) & Préoccupations (eR) \\
\hline 3'50" & Catherine observe les élèves en retrait & $\begin{array}{l}\text { - Sonder l'ambiance générale de la } \\
\text { classe } \\
- \text { Mesurer l'engagement des élèves } \\
\text { dans l'activité }\end{array}$ \\
\hline 3'55" & Catherine se déplace en direction des élèves & $\begin{array}{l}\text { - Rompre le frontal } \\
\text { - Instaurer un climat de confiance }\end{array}$ \\
\hline 4'12" & Catherine se déplace au milieu des élèves & - Rompre le frontal \\
\hline 4'16" & $\begin{array}{l}\text { Catherine se déplace en se mêlant aux } \\
\text { élèves et en suivant les consignes de } \\
\text { l'échauffement }\end{array}$ \\
\hline
\end{tabular}

4. 2. 3. Relations entre les CNV et le contexte de classe : la pertinence des CNV

Du point de vue de l'enseignante, les CNV ont été produites consécutivement à un certain nombre de perceptions de la situation de classe. Son représentamen est : (1) les postures, les choix de trajets, les attitudes des élèves (« je les regarde, comment dire, je regarde si elles sont toutes molles, les mains dans les poches, pas vraiment dedans, ou si elles ont changé de posture» et (2) les élèves «mal à l'aise » dans l'activité. Les CNV ont 


\section{eJRIEPS 37 janvier 2016}

également émergé à partir des connaissances mobilisées par Catherine en situation de classe. Son référentiel a trait : (1) certains élèves n'ont pas un rapport positif à l'APSA ; (2) les élèves peuvent déduire des consignes de l'enseignant que celui-ci ne prend pas part au cours ; (3) il est important de montrer aux élèves que l'enseignant est là pour les aider ; (4) le comportement qui va être produit par les élèves (« il y en a deux je sais qu'elles vont marcher tout le temps côte à côte ») ; (5) l'ambiance de cette classe permet une proximité de l'enseignante et des élèves que d'autres classes ne permettent pas (" il y a des cours, par exemple les secondes, où je ne suis plus du tout dans cette relation là »).

Les perceptions de Catherine lui permettent également de mesurer les effets positifs de ses CNV produites. Son représentamen est : " II se passe quelque chose, il y a une entité là. Et je pense que ça change les choses. Ca change les modes de relation, ça change ". D'après l'analyse extrinsèque de la situation de classe entourant l'étude de cas, nous remarquons que les élèves, qui réalisent des déplacements dans la salle en suivant les consignes de l'enseignante, tendent à converger vers l'enseignante lorsqu'elle prend part à l'échauffement. Malgré tout, il semble difficile d'associer ce mouvement général dans les déplacements des élèves et la proximité soudaine de l'enseignante auprès d'eux.

Finalement, nous constatons que l'analyse extrinsèque ne permet pas de vérifier précisément la perception de l'enseignante selon laquelle sa proximité avec les élèves influe sur l'ambiance et les modes de relation au sein du groupe classe.

4. 2. 4. Analyse des données de l'entretien bilan

L'entretien bilan réalisé avec Catherine met en évidence un certain nombre d'éléments dont certains sont particulièrement en lien avec la question de son engagement en classe, de ce que son corps donne à voir. L'enseignante se dit en effet surprise de ce qu'elle perçoit de son corps en situation de classe: "moi, l'idée que je ressors de cette recherche, c'est de me voir, et de me voir, je me surprends : j'ai l'impression que c'est moi, je me reconnais, mais j'ai l'impression d'être dans un engagement perpétuel, et je me dis que la progression peut-être dans cette intelligence émotionnelle, ça serait d'aller vers une plus grande prise de distance ".

4. 3. Etude de cas $n^{\circ} 3:$ formes et fonctions des CNV de François en cours de arts du cirque (non spécialiste) avec une classe de quatrième

L'étude de cas $n^{\circ} 3$ propose une analyse de l'activité de François lors du premier cours d'arts du cirque d'un cycle de huit cours, avec 17 élèves de première. François a débuté le cours d'arts du cirque par un regroupement des élèves. Debout face aux élèves assis au milieu du gymnase sur un tapis déjà positionné, il a commencé par leur expliquer que 


\section{eJRIEPS 37 janvier 2016}

l'activité cirque faisait partie du programme et que certains d'entre eux vont choisir cette activité pour le baccalauréat. Puis il a plaisanté avec les élèves en sous-entendant que certains étaient déjà des clowns. Suite à cette courte présentation, il a demandé aux élèves de se rassembler devant le tableau afin de rentrer plus précisément dans le fonctionnement du projet de cycle. L'étude de cas $n^{\circ} 3$ se situe entre le moment où François s'est rapproché du tableau et demande aux élèves de se rapprocher également. II poursuit ses explications face aux élèves, toujours debout tandis que les élèves sont assis (de 2'54"' à 4'15').

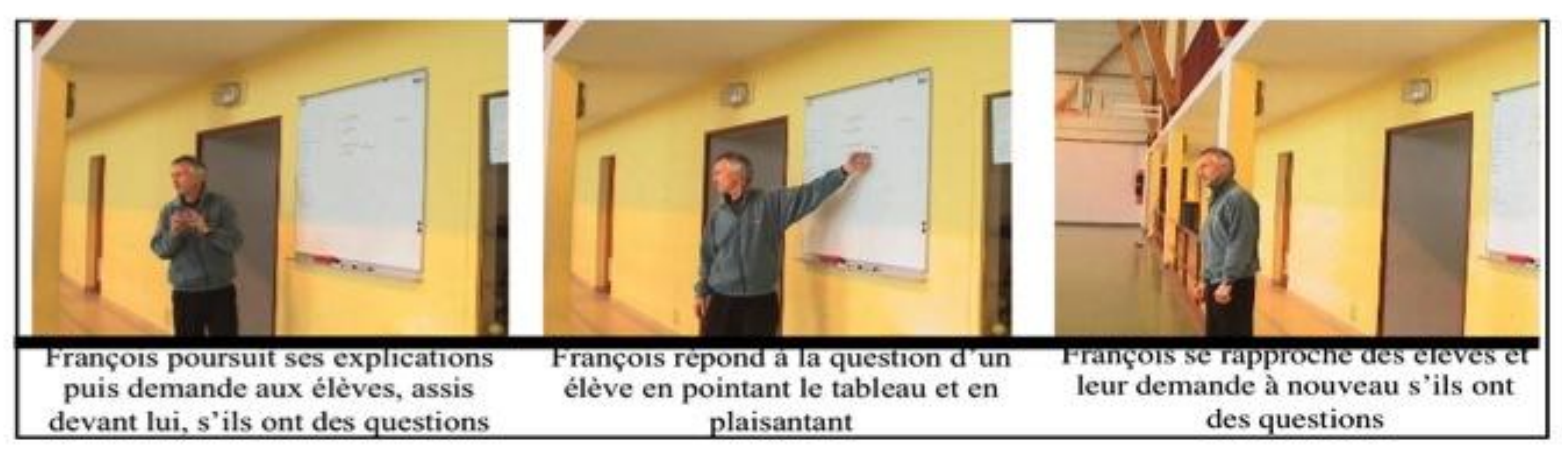

Figure 5. Description de l'activité de François (étude de cas $\mathrm{n}^{\circ} 3$ ).

Dans cette configuration d'activité collective, François est debout devant le tableau, les élèves assis et regroupés devant lui. II alterne des courts déplacements en direction des élèves et en direction du tableau.

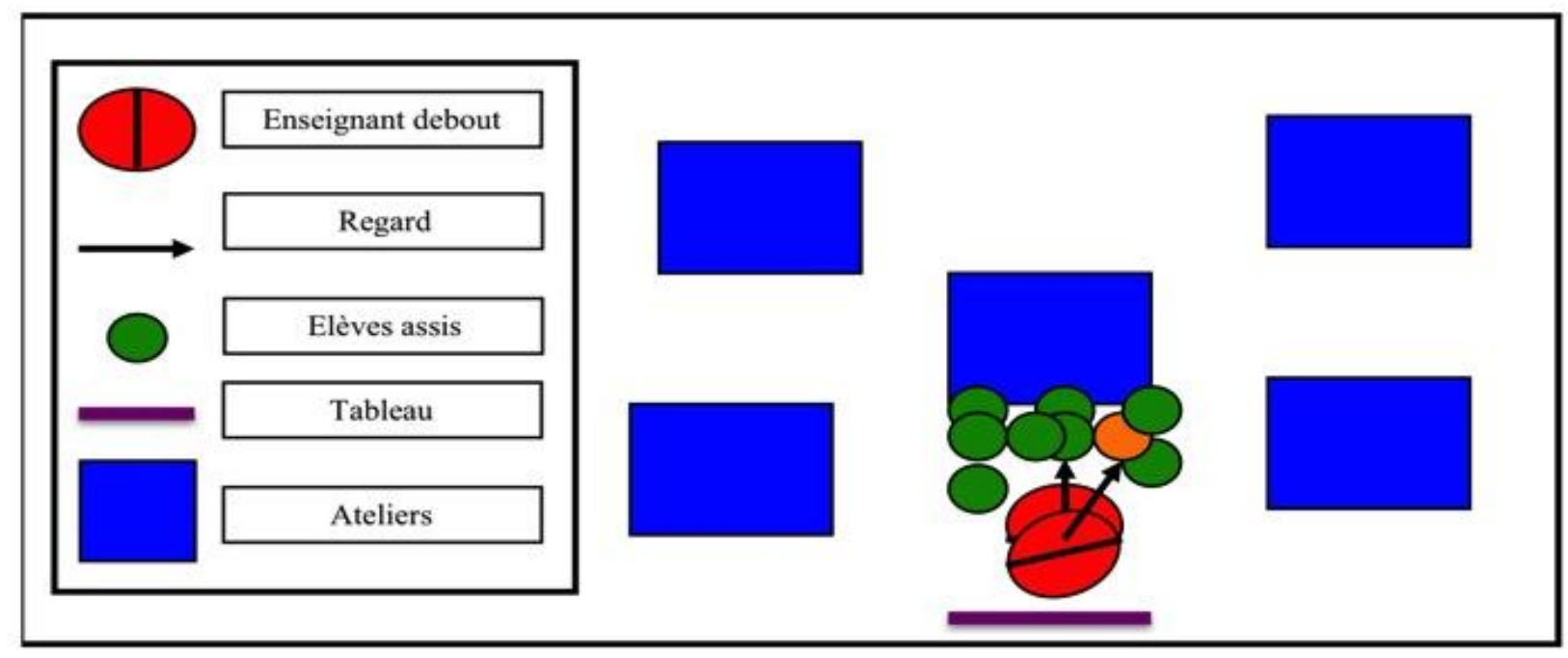

Figure 6. Schéma de la configuration de l'activité collective (étude de cas n`3).

4. 3. 1. Analyse des données d'observation et de verbalisation in situ: les formes de CNV 


\section{eJRIEPS 37 janvier 2016}

Dans un premier temps, François explique aux élèves qu'il vont avoir à se répartir sur des ateliers et à travailler la jonglerie et l'équilibre : "donc en cirque, vous allez travailler sur deux parties. Une partie qui est jonglerie. Deuxième partie, équilibre. Vous avez toutes ces possibilités là. Maintenant, au niveau du programme sur l'ensemble des séances, vous aurez à me montrer deux activités jongleries parmi celle là, deux activités équilibre, et une activité jonglerie et équilibre à deux. Est-ce qu'il y a des questions ? ». Durant ce temps, François produit plusieurs formes de CNV : il réalise des gestes de la main accompagnant ses paroles. Dans un deuxième temps, une élève interroge François concernant un des ateliers : les "bolasses ». II répond à l'élève le corps orienté vers le tableau, en pointant l'atelier concerné sur le tableau : "Les bolasses ? Ou sont les bolasses ? Voilà ! But du jeu, les faire tourner sans s'assommer et sans assommer les autres. Sur les différents ateliers, vous avez des feuilles comme ça qui précisent ce qu'il est possible de faire. Donc on verra en fonction du temps que l'on a, vous avez différents niveaux, et donc ça, ça peut vous guider sur ce qu'il y a à faire. Le but du jeu, bon normalement vous êtes face à un public, c'est de créer de l'émotion. Le public ça sera moi ou ce sera vous, donc essayez de surprendre". Directement après, il sourit et se remet à plaisanter avec les élèves, toujours en pointant le tableau : "donc naturellement je suis émerveillé devant vous, mais là il faudra m'émerveiller un peu plus. C'est bon ? ”. Dans un dernier temps, l'enseignant s'éloigne du tableau pour se rapprocher des élèves et répondre à d'autres questions.

4. 3. 2. Analyse des données de verbalisation a posteriori : les fonctions des CNV

L'entretien d'autoconfrontation nous permet de mettre en évidence les préoccupations de François aux différents temps décrits, qui témoignent de fonctions particulières des CNV du point de vue de l'enseignant. Lorsqu'il poursuit ses explications devant le tableau, il a pour préoccupation de présenter la leçon aux élèves, mais aussi d'affirmer son autorité sur eux : "souvent, je les fais asseoir. Alors inconsciemment, c'est une prise de pouvoir : ils sont en-dessous. En plus, c'est vrai que je ne suis pas grand, et en lycée, ils ont tous une taille de plus que moi ». Paul précise : "ma position fait un petit peu solennel, c'est ma façon d'être aussi : je suis pas leur copain, j'ai une attitude très neutre, une attitude de respect envers le maître. Bon ce temps là, il m'appartient ". Dans ce cas, les CNV de l'enseignant, qui ont pour forme principale la posture debout de l'enseignant face aux élèves assis, ont pour fonction d'affirmer l'autorité de l'enseignant face aux élèves. Dans un deuxième temps, Paul répond à la question d'un élève. Sa préoccupation est alors de sauver la face, de manière à compenser son manque de connaissances dans I'APSA et 


\section{eJRIEPS 37 janvier 2016}

sa difficulté de répondre précisément aux questions : "je ne suis pas mal à l'aise, mais toujours un peu négatif par rapport à l'activité. Mais effectivement, tu vois, le fait de rigoler avec les bolasses, bon c'est un peu pour sauver la face quoi... Autant au badminton, je suis gêné parce que je n'ai pas le temps de leur apporter tout ce que je veux, je sais à un moment donné ce que je leur apporte pas, là la question c'est davantage qu'est-ce que je peux leur apporter. Là, je suis en même temps en train de leur parler, et en même temps je me parle à moi. Il y a un discours interne relativement intense : ou je vais quoi..." ". Dans un dernier temps, les préoccupations de François ont trait à la présentation de la leçon et à la dynamisation de l'activité des élèves (Tableau 3).

Tableau III. Récit réduit du cours d'action de François (étude de cas n³).

\begin{tabular}{lll}
\hline Temps & Unités Elémentaires (UE) & Préoccupations (eR) \\
\hline 2'54" & $\begin{array}{l}\text { François poursuit ses explications puis } \\
\text { demande aux élèves assis devant lui, s'ils ont } \\
\text { des questions }\end{array}$ & - Affirmer son autorité sur les élèves \\
\hline 3'55" & $\begin{array}{l}\text { François répond à la question d'un élève en } \\
\text { pointant le tableau et en plaisantant }\end{array}$ & $\begin{array}{l}\text { - Sauver la face en compensant par } \\
\text { l'humour le manque de connaissances } \\
\text { dans l'APSA }\end{array}$ \\
\hline 4'12" & $\begin{array}{l}\text { François se rapproche des élèves et leur } \\
\text { demande à nouveau s'ils ont des questions }\end{array}$ & - Présenter la leçon \\
\end{tabular}

4. 3. 3. Relations entre les CNV et le contexte de classe : la pertinence des CNV

Du point de vue de l'enseignant, les CNV ont été produites consécutivement à un certain nombre de perceptions de la situation de classe. Son représentamen a exclusivement trait aux questions qui lui sont posées par les élèves. Les CNV ont également émergé à partir des connaissances mobilisées par François en situation de classe. Son référentiel a trait : (1) à sa propre connaissance de l'APSA (« c'est sur que ne pouvant pas montrer ce que l'on peut faire, je détourne avec un peu d'humour. Là, on va rentrer un peu dans mes limites par rapport au cirque »); (2) la qualité de ses relations avec les élèves ("par contre, je sais que je n'ai pas de problème avec les élèves »).

Les perceptions de François lui permettent également de mesurer les effets positifs de ses CNV produites. Son représentamen est: "C'est moi qui parle, il (ce moment là) m'appartient, donc j'ai une attitude... j'ai pas besoin de me mettre en scène. On voit, ils me regardent tous, je n'ai pas à attirer particulièrement l'attention: je sais qu'ils m'écoutent (...) C'est à dire que souvent, on perçoit davantage ceux qui tournent que ceux qui regardent, mais effectivement, je sens que là, si je parle, il n'y a pas de problème ". D'après l'analyse extrinsèque de la situation de classe entourant l'étude de cas, nous constatons en effet qu'un certain nombre de questions sont posées par les élèves, 


\section{eJRIEPS 37 janvier 2016}

notamment une, concernant l'utilisation des bolasses. L'enseignant opte alors pour une réponse humoristique et génère de façon claire des rires chez les élèves. Aucun comportement inattentif ou inapproprié n'est produit lors de ce moment.

Finalement, nous constatons que l'analyse extrinsèque de la situation de classe vient vérifier la perception de François au sujet de ce qui génère ses CNV et des effets positifs de ces dernières sur la situation de classe.

4. 3. 4. Analyse des données de l'entretien bilan

Au cours de l'entretien bilan, François a exprimé son opinion quant aux apports de sa participation à la recherche, insistant notamment sur ce que son corps donne à voir aux élèves, dont il n'avait pas forcément conscience : « je n'ai pas du tout été gêné par le fait de me regarder et puis de voir des choses, et au contraire j'ai trouvé ça super intéressant parce que justement on voit des choses, ben qu'on perçoit pas forcément de manière consciente. Ca je trouve ça, c'est vraiment très intéressant ».

4.4. Etude de cas $n^{\circ} 4$ : formes et fonctions des CNV de Christophe en cours de tennis de table (spécialiste) avec une classe de première

L'étude de cas $n^{\circ} 4$ propose une analyse de l'activité de Christophe lors du troisième cours d'un cycle de dix leçons de tennis de table avec 32 élèves de première. Le cours de tennis de table a débuté avec un rassemblement des élèves. Christophe a commencé par revenir sur la leçon précédente, en insistant sur la nécessité d'exploiter les observations réalisées lors de cette leçon afin d'améliorer l'adaptation du projet tactique aux caractéristiques de l'adversaire. II s'agit également pour les élèves de continuer à s'organiser de façon autonome dans les situations d'apprentissage. Christophe a ensuite fait l'appel et enchainé sur la présentation de la leçon, qui consiste pour les élèves à gagner l'échange au bon moment en fonction de son jeu et surtout de celui de son adversaire. L'étude de cas $n^{\circ} 4$ se situe entre le moment où Christophe a commencé à présenter la situation d'échauffement en mimant un top-spin, et le moment où il se met à démontrer un coupé, alors que les élèves sont assis derrière lui (de 1'56" à 2'52'). 


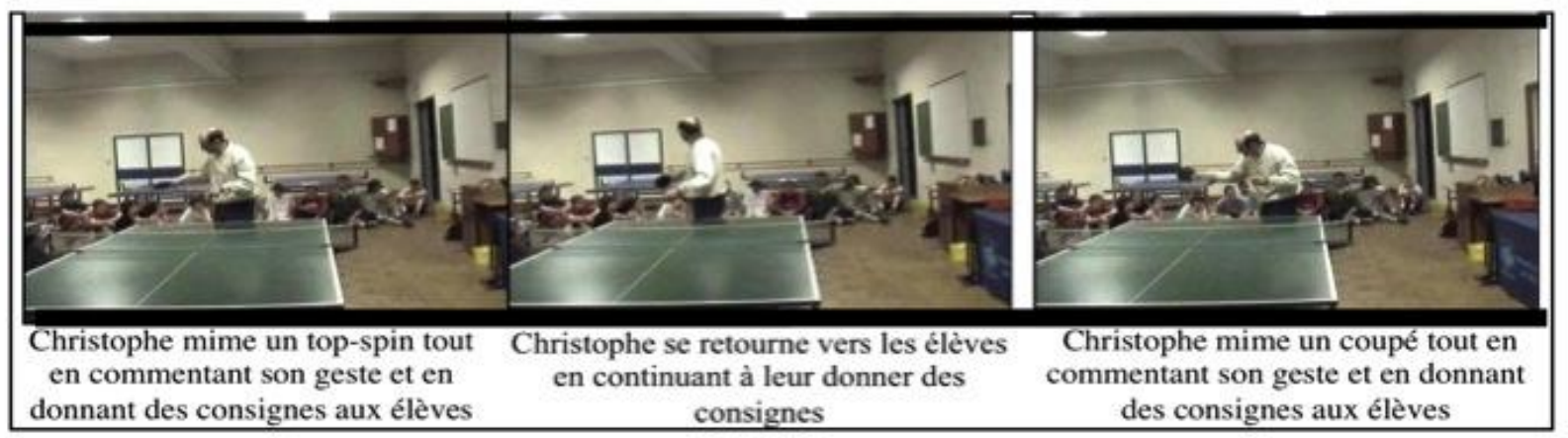

Figure 7. Description de l'activité de Christophe (étude de cas $n^{\circ} 4$ ).

Dans cette configuration d'activité collective, les élèves sont assis et regroupés autour de l'enseignant debout, qui alterne une posture leur tournant le dos lorsqu'il démontre un geste et une posture ainsi qu'un regard plus orienté vers eux (Figure 8).

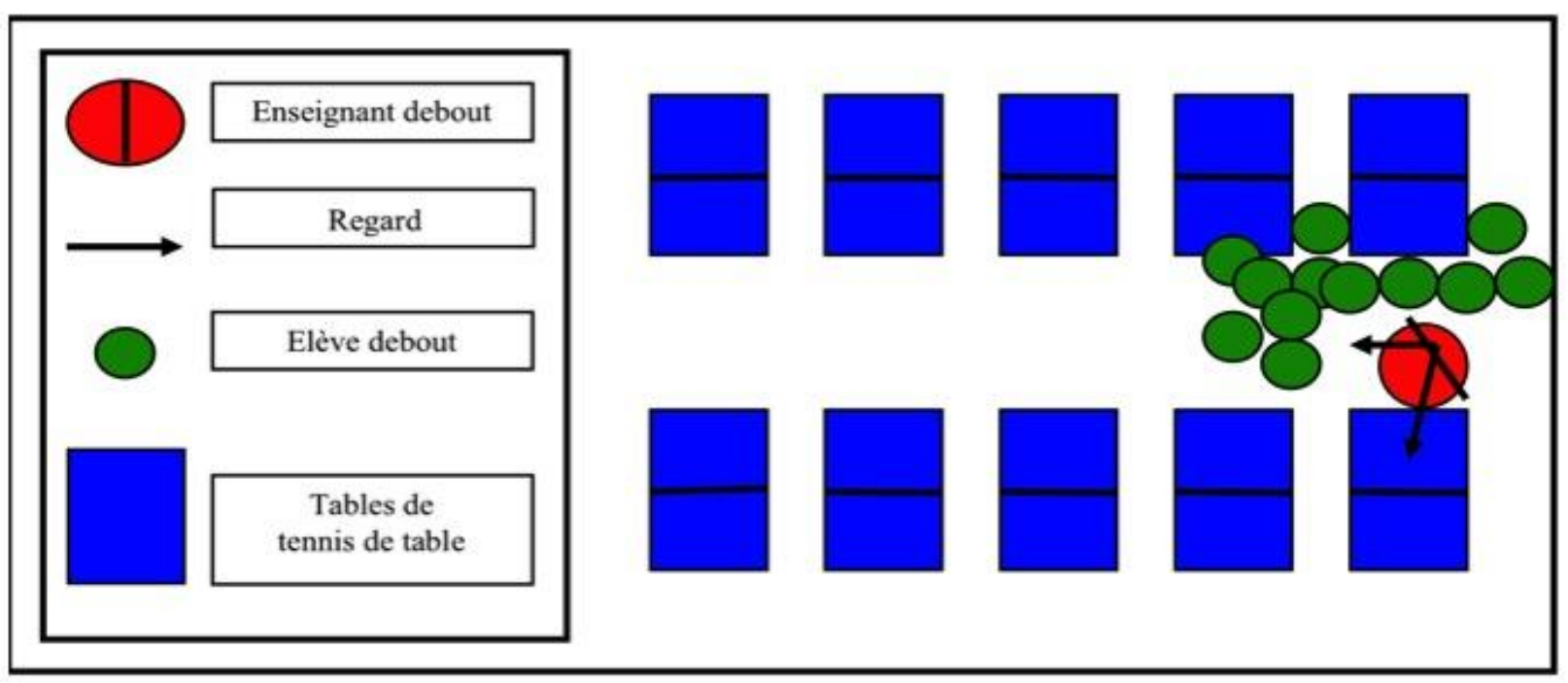

Figure 8. Schéma de la configuration de l'activité collective (étude de cas $\mathrm{n}^{\circ} 4$ ).

4. 4. 1. Analyse des données d'observation et de verbalisation in situ : les formes de CNV

Dans un premier temps, Christophe mime un top-spin tout en commentant ses gestes : " ce que j'aimerais voir : un, se déplacer pour être à bonne distance de frappe. Alors je rappelle que quel que soit ce que je fais comme exercice, c'est pas la raquette que je déplace, c'est mon corps pour être bien situé par rapport à la balle. Par exemple, là à quelle distance ? A demi-bras. Si j'ai une balle en revers, face à la balle». Il est positionné dos aux élèves assis, le corps orienté légèrement de côté de sorte à ce qu'ils puissent voir ses gestes. Dans un deuxième temps, l'enseignant tourne la tête en direction des élèves, tout en poursuivant son intervention verbale: "Donc quelle que soit la situation d'échauffement, vous devez toujours respecter ça ». Dans un troisième temps, Christophe 


\section{eJRIEPS 37 janvier 2016}

mime un coupé, tout en commentant son geste et en donnant des consignes aux élèves : " deuxième thème, c'est calibrer ses frappes, donc ça dépend ce que vous faites : au niveau 1 c'est orienter la raquette vers l'endroit ou je veux diriger la balle, au niveau 2, quand vous êtes plus près de la table, essayer de rabattre la balle le plus possible, et vers le niveau 2, couper en essayant d'accompagner la balle le plus possible. D'accord? Ca dépend de votre niveau. Troisième thème, se préparer au match : dans cette dernière situation, il y en a un qui est serveur et l'autre est renvoyeur. Sur vos feuilles, vous devez marquer à chaque fois le résultat de ce que vous faites ". Dans cette situation, les formes principales de CNV de Christophe ont trait aux gestes représentant des coups en tennis de table (Figure 7).

4. 4. 2. Analyse des données de verbalisation a posteriori : les fonctions des CNV

L'entretien d'autoconfrontation a révélé que l'enseignant avait des préoccupations de plusieurs ordres dans la situation, en lien avec des fonctions particulières des CNV dans la situation. Ces préoccupations sont les mêmes lors des trois temps de la situation : (1) l'utilisation des gestes en tennis de table a selon Christophe la fonction de le faire gagner en crédibilité aux yeux des élèves: "moi j'estime qu'un prof d'EPS doit s'impliquer physiquement à certains moments, pour avoir une certaine crédibilité (...) j'espère qu'il y a une certaine crédibilité par rapport aux connaissances que je peux leur apporter »; (2) les formes de CNV utilisées par Christophe ont aussi pour fonction de présenter clairement aux élèves ce qui est attendu d'eux lors de la leçon : "la présentation des objectifs et la première séquence, pour moi, c'est un moment clef de la séance "; (3) Christophe souhaite également, dans la situation, influencer l'ambiance de classe de sorte que les élèves travaillent sérieusement tout en prenant du plaisir à pratiquer l'activité : « moi ce que je recherche, c'est qu'il y ait à la fois du plaisir, c'est à dire qu'ils rentrent dans cette séance là en ayant du plaisir à pratiquer l'activité duelle, donc sous forme de défi, de situation record... Une deuxième notion importante, qu'ils rentrent dans une démarche d'apprentissage. Donc à la fois, je me met en projet pour éprouver du plaisir dans l'activité, et également, je suis là pour apprendre, je vais prendre du plaisir à apprendre, il y a les deux choses qui se combinent 》 (Tableau 4).

Tableau IV. Récit réduit du cours d'action de Christophe (étude de cas $n^{\circ} 4$ )

\begin{tabular}{llr}
\hline Temps & Unités Elémentaires (UE) & Préoccupations (eR) \\
\hline $1 ' 56 "$ & $\begin{array}{l}\text { Christophe mime un top-spin tout en - Asseoir sa crédibilité face aux élèves } \\
\text { commentant son geste et en donnant des } \\
\text { consignes aux élèves a la réalisation des gestes }\end{array}$ \\
\hline 2'17" & Christophe se retourne vers les élèves en aux élèves en préfigurant les objectifs \\
\hline
\end{tabular}




\begin{tabular}{ll}
\hline continuant à leur donner des consignes & de la leçon \\
\hline 2 '29" & $\begin{array}{l}\text { Christophe mime un coupé tout en - Instaurer une ambiance de travail à la } \\
\text { commentant son geste et en donnant des fois plaisante et studieuse } \\
\text { consignes aux élèves }\end{array}$
\end{tabular}

4. 4. 3. Relations entre les $\mathrm{CNV}$ et le contexte de classe : la pertinence des CNV

Du point de vue de Christophe, les CNV ont été produites principalement au regard de ses connaissances mobilisées en situation de classe. Son référentiel a trait à sa connaissance de l'APSA tennis de table : « alors je le fais pas systématiquement (démontrer des gestes) dans toutes les APS mais comme en tennis de table j'en ai fait en club, que lors de séances précédentes, je leur ai montré certains aspects techniques, je peux me permettre d'illustrer sans adversaire en face ».

Les perceptions de Christophe lui permettent de mesurer les effets positifs de ses CNV produites. Son représentamen est : « là j'ai l'impression qu'il ont été plutôt en écoute ».

D'après l'analyse extrinsèque, nous constatons que les regards des élèves sont tournés vers l'enseignant, que les élèves ne produisent aucun comportement déviant et que le silence règne lors des explications de Christophe.

Finalement, nous constatons que l'analyse extrinsèque de la situation ne permet pas de déterminer ce qui génère les gestes de l'enseignant spécifique à l'APSA. Celui-ci semble davantage s'appuyer sur ses connaissances liées à son expérience de spécialise dans I'APSA que sur des signes perçus en situation de classe. En outre, l'analyse extrinsèque des effets de cette intervention entre en concordance avec les perceptions de l'enseignant concernant le niveau d'écoute des élèves.

4. 4. 4. Analyse des données de l'entretien bilan

L'entretien bilan réalisé avec Christophe montre que lui aussi retient de la recherche le fait de s'être vu en vidéo, notamment parce que les élèves perçoivent l'enseignant et ce que son corps donne à voir. Il est alors nécessaire de développer un certain charisme : « je pense que finalement, on ne leurre pas les élèves. C'est à dire que les élèves te perçoivent bien tel que tu es, sur la distance quoi. Surtout si tu les as sur plusieurs années, ils te perçoivent, même au niveau émotionnel. Moi personnellement ça m'a aidé parce que par exemple, ce côté charismatique que j'avais entre guillemets, perdu je pense, j'essaie de le remettre en œuvre. Ca m'a aidé là dessus».

4. 5. Etude de cas $n^{\circ} 5$ : formes et fonctions des CNV de Philippe en cours de danse (non spécialiste) avec une classe de sixième. 


\section{eJRIEPS 37 janvier 2016}

L'étude de cas $n^{\circ} 5$ propose une analyse de l'activité de Philippe lors de la troisième leçon d'un cycle de dix leçons en Danse, avec une classe de sixième SEGPA composée de 19 élèves. Le cours a débuté par un regroupement des élèves devant l'enseignant. Philippe a décidé en premier lieu de revenir sur les comportements inappropriés des élèves lors du trajet menant à l'installation sportive. En effet, les élèves ont profité du trajet pour chahuter, jouer avec des canettes, crier, lancer des injures, etc. L’étude de cas $n^{\circ} 5$ se situe entre le moment où Philippe regroupe les élèves et les interpelle sur leur comportement lors du trajet, et le moment où il se met à lister les différents points de mécontentement (de 28"' à 1'14").

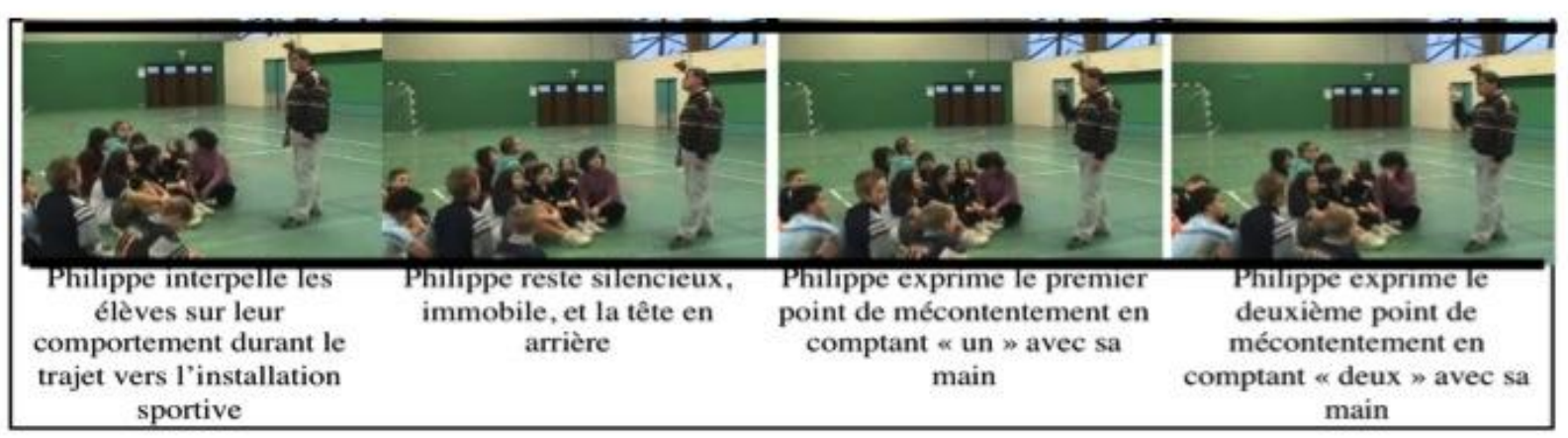

Figure 9. Description de l'activité de Philippe (étude de cas $n^{\circ} 5$ ).

Dans cette configuration d'activité collective, l'enseignant et les élèves se trouvent regroupés au centre de la salle. L'enseignant est debout face aux élèves regroupés devant lui (Figure 10).

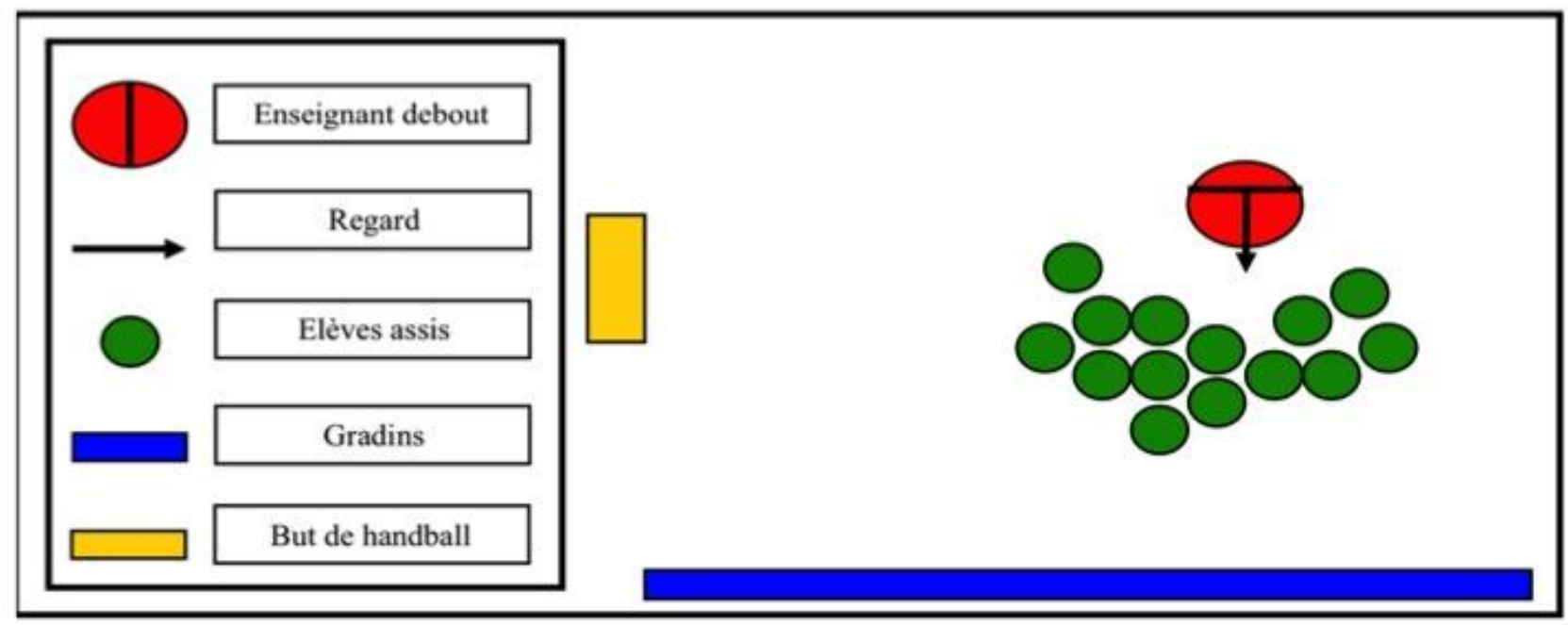

Figure 10. Schéma de la configuration de l'activité collective (étude de cas n5). 


\section{eJRIEPS 37 janvier 2016}

4. 5. 1. Analyse des données d'observation et de verbalisation in situ: les formes de CNV

Dans un premier temps, Philippe exprime aux élèves qu'il n'est pas content de leur comportement sur le trajet menant à l'installation sportive : " avant que je vous explique le déroulement de la séance, je vais vous dire que déjà, depuis le collège, j'aurai pu crier au moins sept ou huit fois... ". II accompagne cette CV de plusieurs formes de CNV : une posture très droite, une orientation du corps face aux élèves, les bras le long du corps, le regard dirigé vers les élèves, et aucun mouvement des membres inférieurs. Dans un deuxième temps, Philippe marque un silence relativement long, conservant sa posture droite et levant légèrement la tête de sorte à ce que sa posture soit plus droite encore. Dans un troisième temps, Philippe reprend la parole, signifiant aux élèves un premier point de mécontentement: "premièrement parce que vous n'étiez pas en face de la conciergerie pour certains ». II accompagne ces mots en comptant « un " sur ses doigts, et en produisant les mêmes formes de CNV. Dans un dernier temps, Philippe signifie aux élèves un deuxième point de mécontentement, comptant "deux " sur ses doigts et conservant une posture très droite : "deuxièmement parce qu'en venant au gymnase, il y en a qui ont joué avec des bouteilles vides sous le hangar vert » (Figure 9).

4. 5. 2. Analyse des données de verbalisation a posteriori : les fonctions des CNV

Les données d'autoconfrontation nous permettent de mettre en évidence plusieurs préoccupations de Philippe lors de la situation. Dans un premier temps, l'enseignant utilise notamment les CNV pour montrer qu'il est attentif aux comportements des élèves et qu'il a perçu ces comportements lors du trajet : "là, je veux leur montrer tout de suite que j'ai tout vu (...) mais surtout leur montrer que je suis attentif à ce qu'ils font ». Au même moment, Philippe souhaite montrer aux élèves qu'il n'est pas content, il cherche à leur communiquer directement son ressenti : "je veux leur montrer que je ne suis pas content». Aussi, Philippe nous dit que la fonction de son intervention, et notamment des $\mathrm{CNV}$, est de cadrer les élèves dès le début, en prévision de la suite du cours : « en fait, je me sers de ce qui s'est passé sur le trajet pour pouvoir les recadrer pour la séance ». A partir du deuxième temps, les CNV produites par Philippe ont pour fonction d'exacerber son mécontentement devant les élèves. Finalement, l'enseignant joue avec ses émotions pour obtenir un effet plus marquant sur eux : «je ne suis pas du tout énervé, c'est plutôt comme un jeu... Bon je suis pas très content quand même de ce qui s'est passé. Mais en fait, c'est plutôt amusant. Le truc, c'est de réussir à être expressif tout en étant calme à 


\section{eJRIEPS 37 janvier 2016}

l'intérieur. Mais j'y pense pas sur le moment, c'est un truc classique pour moi. J'ai fait ça des dizaines et des dizaines de fois. Je veux aussi leur dire franchement mon ressenti ».

Tableau V. Récit réduit du cours d'action de Philippe (étude de cas n5).

\begin{tabular}{|c|c|c|}
\hline Temps & Unités Elémentaires (UE) & Préoccupations (eR) \\
\hline $28 "$ & $\begin{array}{l}\text { Philippe interpelle les élèves sur leur } \\
\text { comportement durant le trajet vers } \\
\text { l'installation sportive }\end{array}$ & $\begin{array}{l}\text { - Montrer aux élèves qu'il a perçu leurs } \\
\text { comportements inappropriés sur le } \\
\text { trajet } \\
\text { - Cadrer les élèves } \\
\text { - Manifester aux élèves son } \\
\text { mécontentement }\end{array}$ \\
\hline $57 "$ & $\begin{array}{l}\text { Philippe reste silencieux, immobile, et la tête } \\
\text { en arrière }\end{array}$ & $\begin{array}{lccc}\text { S'amuser à } & \text { exacerber } & \text { son } \\
\text { mécontentement devant les élèves } & \\
\end{array}$ \\
\hline 1'09" & $\begin{array}{l}\text { Philippe exprime le premier point de } \\
\text { mécontentement en comptant « un » avec sa } \\
\text { main }\end{array}$ & $\begin{array}{l}\text { - Montrer aux élèves qu'il a perçu leurs } \\
\text { comportements inappropriés sur le } \\
\text { trajet } \\
\text { - S'amuser à exacerber son } \\
\text { mécontentement devant les élèves }\end{array}$ \\
\hline 1'12"' & $\begin{array}{l}\text { Philippe exprime le deuxième point de } \\
\text { mécontentement en comptant « deux " avec } \\
\text { sa main }\end{array}$ & $\begin{array}{l}\text { - Montrer aux élèves qu'il a perçu leurs } \\
\text { comportements inappropriés sur le } \\
\text { trajet } \\
\text { - S'amuser à exacerber son } \\
\text { mécontentement devant les élèves }\end{array}$ \\
\hline
\end{tabular}

4. 5. 3. Relations entre les CNV et le contexte de classe : la pertinence des CNV

Du point de vue de Philippe, il ressort que les perceptions liées aux comportements déviants des élèves, notamment sur le trajet menant au gymnase, l'amènent à produire ces CNV en situation de classe. Ces CNV ont également été produites en lien avec ses connaissances mobilisées en situation de classe. Son référentiel a trait : (1) à l'importance de cadrer les élèves dès le début de la leçon ; (2) au fait que jouer avec ses émotions, théâtraliser son intervention, fonctionne bien avec ces élèves (« je sais aussi que ce type d'intervention, ça marche bien avec ces classes, avec les élèves de mon collège »).

Les perceptions de Philippe lui permettent également de mesurer les effets positifs de ses CNV produites. Son représentamen est principalement l'impact qu'il a sur les élèves (« disons que je sens si ça marche ou pas... Là, j'avais l'impression d'avoir un certain impact, donc je continue »).

D'après l'analyse extrinsèque de la situation de classe entourant l'étude de cas, nous remarquons que les élèves ont produit sur le trajet plusieurs comportements déviants. Suite à l'intervention de Philippe, au cours de laquelle il a produit ces CNV, les élèves ne témoignaient d'aucun comportement inattentif. Les regards étaient portés sur l'enseignant, aucun bavardage n'a été recensé et un silence prolongé régnait en classe. 


\section{eJRIEPS 37 janvier 2016}

Finalement, nous constatons que les perceptions de Philippe, notamment en ce qui concerne l'impact de son intervention sur les élèves, ont été vérifiées par l'analyse extrinsèque de la situation de classe.

\section{5. 4. Analyse des données de l'entretien bilan}

De manière très explicite, Philippe exprime durant l'entretien bilan la surprise qu'il a ressentie en se voyant à la vidéo. Cette surprise provient du décalage entre ce qu'il pensait donner à voir corporellement aux élèves et ce qu'il donne effectivement à voir, d'après lui, sur la vidéo: "le fait de se voir, de se percevoir parfois différemment de ce que l'on pense (...) l'image que je donne de moi aux élèves, et que je perçois là, qui n'est pas tout à fait en adéquation avec l'image que j'ai de moi-même face aux élèves. Ca c'est un point énorme, super intéressant. En ce qui me concerne, je trouve que je suis très crispé, quand je me vois. C'est pas ce que je vis intérieurement. Je ne suis pas crispé quand j'enseigne Par contre je suis concerné, tout le temps ».

\section{Discussion}

Les résultats sont discutés en deux parties: (1) la complexité des relations entre les formes et les fonctions des CNV des enseignants experts en EPS et leurs CV en situation de classe ; (2) les relations entre les CNV et le contexte de classe : la pertinence des CNV.

5. 1. La complexité des relations entre les formes et les fonctions des CNV des enseignants experts en EPS et leurs CV en situation de classe

En premier lieu, nos résultats mettent particulièrement en évidence la complexité des formes des CNV dans l'activité des enseignants d'EPS experts en situation de classe, qui sont souvent imbriquées et difficilement séparables. Dans le cas de Paul, qui a pour préoccupation principale de recadrer un élève, nous constatons que la CNV regroupe un regard appuyé sur lui, un bras le pointant, une orientation du corps exprimant un face à face avec lui, une position debout associée à une posture droite face à cet élève assis au sein du groupe. Si les différentes catégories de formes, mises en évidence notamment par Moulin (2004) et par Boizumault et Cogérino (2012), se retrouvent dans notre étude, reste qu'elles y sont décrites comme profondément enchâssées et inséparables. Cette indissociabilité des différentes formes de CNV entre en cohérence avec une conception " orchestrale " de la communication, qui considère celle-ci comme un système auquel l'acteur social participe à tout instant, par ses gestes, son regard, son silence, sinon son absence, et non comme une grammaire de gestes (Winkin, 1981). 


\section{eJRIEPS 37 janvier 2016}

En deuxième lieu, concernant les fonctions des CNV, nous constatons que l'enseignant est mû par des préoccupations multiples et imbriquées lorsqu'il produit en classe des formes particulières de CNV. Aux fonctions des CNV liées à l'apprentissage des élèves, mises en évidence notamment par Mahut et al. (2005) et par Forest (2008), s'additionnent d'autres fonctions liées par exemple à la recherche de crédibilité de l'enseignant. En effet, lorsque Christophe réalise des gestes spécifiques au tennis de table, il a non seulement la préoccupation de rendre ses propos intelligibles aux élèves pour favoriser leurs apprentissages, mais aussi de gagner en crédibilité en témoignant d'une connaissance précise de I'APSA. En outre, dans d'autres situations au cours desquelles l'enseignant exprime la préoccupation majeure de cadrer les élèves, comme c'est le cas chez Paul et Philippe, les préoccupations d'apprentissage ne sont pas pour autant absentes. Paul dit en effet que cadrer les élèves est nécessaire pour gagner du temps et ainsi mettre en place les conditions favorables aux apprentissages. Dès lors, les CNV comportent, d'après les préoccupations des enseignants, des fonctions enchâssées qui évoluent et incluent, parfois au premier plan, parfois au second plan, la question de l'apprentissage, de sorte que ce dernier n'est jamais totalement absent ni exclusif.

Par ailleurs, il nous paraît nécessaire de nuancer les relations entre les formes et les fonctions des CNV des enseignants en classe. A l'instar de Kammoun et Amade-Escot (2007), certaines typicalités apparaissent dans les relations entre les formes et les fonctions des CNV des enseignants participants à notre étude. Par exemple, Paul, Philippe et François, qui cherchent à asseoir leur autorité et à cadrer les élèves, optent tous pour des formes semblables de CNV (silences prolongés, regard fixe sur les élèves, posture droite...). Toutefois, il nous paraît difficile de relier de manière stricte certaines catégories de formes à des fonctions particulières. Par exemple, Christophe, qui cherche lui aussi à affirmer son autorité en classe en gagnant en crédibilité face aux élèves, opte pour des formes de CNV liées à des gestes techniques spécifiques à l'APSA. II apparaît dès lors que les formes et les fonctions des CNV sont profondément liées à la singularité des enseignants experts et du contexte. Dit autrement, en nous inspirant de l'idée de Clot (1999) en psychologie du travail, s'ils utilisent un "genre » commun face aux élèves (affirmer leur autorité), reste qu'ils s'approprient ce " genre » pour développer un " style » singulier dans les formes et les fonctions de leurs CNV en classe.

Cette étude a par ailleurs permis d'approfondir l'analyse des complémentarités entre les CNV et les CV de l'enseignant en situation de classe. Nous constatons en effet que les CNV produites par l'enseignant peuvent être utilisées comme un support aux CV, 


\section{eJRIEPS 37 janvier 2016}

notamment lorsque Paul recadre un élève en le désignant par les mots et par le geste. Par contre, dans l'étude de cas analysant l'activité de Catherine, les CNV sont utilisées de manière à se substituer à la CV : l'enseignante se mêle aux élèves pour créer un rapprochement avec eux et favoriser leur engagement, sans leur expliciter cette préoccupation. Un lien peut alors être effectué entre ces résultats et les relations entre CV et CNV selon Argyle (1972). Les deux premières catégories de résultats semblent en effet apparaître dans l'analyse des CNV produites par les enseignants. En ce qui concerne la dernière catégorie, liée aux attitudes, émotions et manipulations de la situation sociale immédiate, il nous semble qu'elle peut être considérée comme contenue dans les deux premières. Par exemple, lorsque Philippe simule une colère tout en exprimant verbalement aux élèves son mécontentement, les manipulations et émotions exprimées font office de support de la CV.

En définitive, il nous semble que cette complexité des relations entre les formes et les fonctions des CNV des enseignants, en lien avec leurs CV, les apparente à de véritables acteurs de théâtre (Pujade-Renaud, 1983 ; Runtz-Christian, 2000). Ce résultat s'illustre à travers l'activité de Philippe, qui, en comptant sur ses doigts les comportements déviants des élèves, exacerbe volontairement sa colère. Un certain décalage existe alors entre ce que l'enseignant donne à voir et ses préoccupations réelles. Dans ce cas, Philippe « habite son corps » et exprime une qualité de présence, une pensée qui se transmet par un corps qui ne le contredit pas, caractéristique, selon Varela (1996), de l'expertise. Visioli et al. (2014) ont montré à ce titre comment les enseignants d'EPS experts parvenaient à jouer avec leurs émotions, à l'aide des gestes ou encore du regard, afin d'obtenir différents effets sur les élèves. A l'inverse, Ria, Saury, Sève et Durand (2001) ont établi que le corps des novices pouvait aller jusqu'à les trahir en situation de classe, révélant aux élèves leurs émotions contradictoires. Nos résultats vont aussi dans le sens d'un rôle important de l'expertise dans la manière d'utiliser le corps en classe. Cette expertise ne se limite pas à une spécialisation dans l'APSA. En effet, François, qui n'est pas spécialiste des arts du cirque, se retrouve en difficulté et parvient à utiliser habilement l'humour (sourire, posture relâchée) de manière à sauver la face, mais aussi à dynamiser l'activité des élèves. Cette situation révèle la capacité de l'enseignant à moduler son intervention et à s'adapter au contexte, en dépit d'un manque de connaissances dans l'APSA. Si l'expertise doit davantage être considérée comme dépendante des situations que comme une propriété stable (Saury, Sève, Leblanc \& Durand, 2002), elle ne peut dès lors pas être restreinte à une unique connaissance du contenu disciplinaire. 


\section{eJRIEPS 37 janvier 2016}

5. 2. Relations entre les CNV et le contexte de classe : la pertinence des CNV Les résultats obtenus au cours de cette recherche montrent que les formes et les fonctions des CNV sont fondamentalement situées et présentent ainsi une certaine pertinence en classe. Bien qu'une approche par études de cas n'ambitionne pas d'accéder à des généralisations, il semble ressortir que ces formes et ces fonctions des CNV diffèrent selon le contexte temporel du cours et la configuration d'activité collective en classe.

Tout d'abord, nous constatons que les études de cas, sélectionnées à partir du point de vue des enseignants, présentes des formes et des fonctions de CNV produites en début de cours. Si nous ne pouvons en tirer de conclusion générale, reste que le début de la leçon semble un moment crucial, comme l'a mis en évidence Gal-Petitfaux (2015) en analysant l'activité d'enseignants chevronnés. L'auteure montre que pour certains enseignants, il s'agit de gagner l'adhésion des élèves et construire une ambiance collective de travail. Dans notre étude, ces préoccupations se retrouvent chez Paul et Philippe, dont les CNV ont pour fonction principale de cadrer les élèves pour lancer une dynamique de classe favorable pour la suite de la leçon. Gal-Petitfaux (2015) a également montré que dans certains contextes, une des préoccupations typiques des enseignants chevronnés était de profiter du début du cours pour instruire les élèves dès leur arrivée et leur transmettre des explications riches sur la leçon et l'échauffement. Cette préoccupation s'accompagne dans son étude d'un ton solennel de l'enseignant, d'une intonation et d'une posture qui signifient l'exigence d'une l'écoute. Ce résultat transparait également dans notre recherche, notamment à travers l'activité de Christophe, qui se centre dès la prise en main des élèves sur le contenu à enseigner par l'intermédiaire de gestes spécifiques à I'APSA. Ces divergences de préoccupations sont intimement liées au contexte de classe, Paul et Philippe enseignant en collège difficile, et Christophe faisant face à des élèves de première témoignant, dès le début du cours, de préoccupations davantage liées à l'apprentissage.

Par ailleurs, nous constatons que les formes et les fonctions des CNV sont influencées par la configuration de l'activité collective en classe. Lors des regroupements au cours desquels l'enseignant fait face à tous les élèves, comme c'est le cas chez Paul, Philippe, François et Christophe, les formes de CNV produites ont notamment pour fonction d'asseoir l'autorité de l'enseignant. Par contre, lorsque les élèves sont en activité, dans l'étude de cas rapportant la situation vécue par Catherine, l'enseignante utilise les CNV pour témoigner sa proximité avec les élèves et se positionner au même niveau qu'eux. 


\section{eJRIEPS 37 janvier 2016}

Notre étude permet ainsi de montrer que l'activité de l'enseignant, et en particulier ses CNV produites en classe, sont indexées aux configurations d'activité collective, dans la lignée des travaux déjà réalisés à ce sujet dans le cadre du cours d'action (Veyrunes, 2012 ; Vors \& Gal-Petitfaux, 2011).

L'étude de la pertinence des CNV a également été entreprise à travers cette recherche, à partir d'une évaluation des éléments amenant l'enseignant à produire des CNV (contraintes extrinsèques), mais aussi l'impact de ces CNV sur la situation (effets extrinsèques).

Concernant les contraintes extrinsèques, nous constatons que les enseignants ont des perceptions très fines de la situation de classe et des comportements des élèves, qui participent de la pertinence des CNV. C'est le cas de Catherine qui perçoit les postures, les choix de trajets, les attitudes des élèves mais aussi l'état émotionnel de ces derniers, concluant que certains sont mal à l'aise dans l'activité. Ce sont ces perceptions qui l'amènent à se rapprocher des élèves de manière à instaurer une relation de proximité favorable à leur engagement. Cizeron et Gal-Petitfaux (2005) ont également mis en évidence cette finesse des perceptions chez des enseignants expérimentés en gymnastique, qui parviennent à interpréter rapidement les formes de corps perçues chez les élèves. La pertinence de la mobilisation des CNV tient également aux connaissances des experts, particulièrement variées et contextualisées (Rovegno, 2007). Par exemple, une des connaissances mobilisées par Catherine lorsqu'elle choisit de se mêler aux élèves pour leur signifier qu'elle travaille avec eux et ne se contente pas d'énoncer des consignes, a trait aux différences d'ambiance entre ses classes : la proximité instaurée avec les élèves diffère d'une classe à l'autre.

Compte tenu que "le plus difficile est de parvenir à se représenter l'effet d'un geste ou d'une parole sur le corps de l'enseigné » (Andrieu, 2003, p. 9), nous avons également cherché à mesurer les effets extrinsèques des CNV des enseignants sur la situation de classe, notamment l'activité des élèves. II ressort que, dans le cas de Philippe, de Paul et de François, le représentamen est notamment l'impact de leur intervention sur les élèves. Ces effets sont confirmés par l'analyse extrinsèque du comportement des élèves, notamment chez Philippe. Lors de son intervention, un silence règne en classe et les regards des élèves sont fixés sur lui. Des études récentes ont d'ailleurs montré que les élèves percevaient les regards, les mimiques et les gestes des enseignants et qu'ils pouvaient jouer un rôle sur leur activité, en favorisant leur motivation et leur concentration en classe (Zeki, 2009). Dans une recherche analysant l'activité d'enseignants de science 


\section{eJRIEPS 37 janvier 2016}

exerçant dans le secondaire, Azian, Raof, Ismail et Hamzah (2013) ont quant à eux établi qu'il existait une haute congruence entre les formes de CNV produites par les enseignants et celles perçues par les élèves.

Néanmoins, dans une perspective didactique et à partir d'une méthodologie plus qualitative et située, Mahut et al. (2005) ont révélé qu'une partie non négligeable des gestes iconiques de l'enseignant en natation ne trouvait pas de résonance chez l'étudiant. Les auteurs ont évoqué un fort décalage des interprétations de signes entre producteur et interprétant du langage véhiculant les contenus d'enseignement. Cet écart dans l'espace sémantique entre enseignant et étudiant était réduit lorsque ce dernier avait suivi les enseignements de façon régulière. Ramené à notre étude, si les CNV des enseignants experts semblent si intelligibles pour les élèves, c'est sans doute qu'ils ont construit, lors des précédents cours avec l'enseignant, une culture commune permettant de donner sens aux CNV. Plus encore, nous faisons l'hypothèse que la pertinence des CNV, mise en évidence à travers leurs effets sur les élèves, tient notamment à l'expérience étendue qu'ont ces derniers des différents environnements d'apprentissage (Fraser, 2001).

\section{Conclusion}

Outre les éléments discutés précédemment, cette étude permet d'envisager des pistes de réflexion concernant les CNV des enseignants en situation de classe, notamment en termes de perspectives de formation et de recherche. Les résultats de notre étude invitent notamment à interroger la place du corps de l'enseignant dans les formations (Burel \& Andrieu, 2014). En accord avec nos résultats, il s'agirait d'une part de prendre en considération que pour l'enseignant d'EPS, la dimension corporelle occupe une place importante dans la réalisation des gestes de métier dont l'efficacité relève parfois de techniques complexes, incorporées et implicites (Laurent \& Saujat, 2014). Plus que d'établir une grammaire des gestes, dont nous pourrions craindre un caractère prescriptif et décontextualisé, il s'agirait alors d'accorder une place majeure à la pertinence des CNV effectivement produites dans l'activité des enseignants en situation de classe. Nous rejoignons ici les propositions de Roche et Gal (2014) qui invitent en formation à l'exploitation de vidéos afin d'accéder aux dimensions corporelles et signifiantes des habiletés de l'enseignant grâce à la combinaison des ressources de formation.

Les résultats présentés à travers cette étude nous amènent également à formuler deux perspectives de recherche majeures. Une première perspective consisterait à approfondir la notion de pertinence des CNV en situation de classe. D'une part, nous pourrions analyser plus précisément les éléments pris en compte par l'enseignant pour produire des 


\section{eJRIEPS 37 janvier 2016}

CNV particulières, notamment les CNV des élèves. D'ailleurs, Vors et Gal-Petitfaux (2014) se sont intéressés à la question de la sensorialité des enseignants, révélant la diversité et la richesse des éléments pris en compte par ce dernier dans l'interaction avec les élèves. Une description fine des CNV des élèves, potentiellement perçues par l'enseignant, pourrait alors permettre de mieux comprendre son activité en acte. D'autre part, concernant les effets des CNV produites par l'enseignant, il s'agirait d'accentuer le caractère collectif de l'analyse des CNV en incluant le point de vue des élèves en situation. Comme l'ont montré Mahut et al. (2005), l'intelligibilité des CNV ne va pas de soi et analyser le cours d'expérience des élèves pourrait alors nous permettre d'évaluer plus précisément les effets des CNV des enseignants sur leur activité.

Une autre perspective pourrait consister à étudier le caractère non significatif pour les enseignants de leurs CNV produites en classe. Nous pourrions analyser le cours d'information des enseignants, qui intègre des éléments ressortant du corps, de la situation et de la culture qui ne donnent pas lieu à expérience, mais qui sont néanmoins pertinents pour l'organisation interne de son activité (Theureau, 2006). Cette perspective paraît d'autant plus intéressante que d'après les données recueillies lors de l'entretien bilan, les enseignants ont exprimé un décalage entre ce qu'ils pensaient donner à voir aux élèves et la réalité de ce qu'ils leur donnent à voir. Ce résultat recoupe ceux de Laurent et Saujat (2014) qui insistent sur l'idée que les gestes de l'enseignant sont des habiletés motrices complexes reposant pour partie sur des automatismes sortis de la sphère des processus conscientisés. Nous pouvons faire l'hypothèse qu'étudier les CNV non significatives des enseignants experts pourrait permettre de révéler certaines compétences de communication incarnées, construites au fil de l'expérience professionnelle. Nous pourrions alors mieux comprendre comment, dans l'enseignement et plus généralement "dans l'écoulement de la vie courante, le corps s'évanouit. Infiniment présent puisqu'il est le support inévitable, la chair de l'être-au-monde de l'homme, il est aussi infiniment absent à sa conscience »(Le Breton, 2000, p. 126).

\section{Bibliographie}

Andrieu, B. (2003). Corps, peau, silences, dans l'enseignement. Nancy : CRDP Lorraine. Argyle, M. (1972). Non-verbal communication in human social interaction. In R. A. Hinde (Ed.), Non-verbal Communication. Cambridge university press, 243-269.

Austin, J. (1970). Quand dire, c'est faire. Paris : Seuil. 


\section{eJRIEPS 37 janvier 2016}

Azian, A. A., Raof, A. H. A., Ismail, F. \& Hamzah, M. (2013). Communication strategies of non-native speaker novice science teachers in second language science classrooms, System, 2, 41, 283-297.

Barbier, J.M. \& Durand, M. (2003). L'activité : un objet intégrateur pour les sciences sociales ? Recherche et Formation, 42, 99-117.

Barbier, J.M. \& Durand, M. (2006). Les rapports sujets - activités - environnements. Approches transverses. Paris : PUF.

Bateson, G. (1984). La nature et la pensée. Paris : Seuil.

Birdwhistell, R.L. (1974). The langage of the Body. In A. Silverstein (Ed.), Human Communication : Theoretical Explorations. New York : Wiley.

Boizumault, M. \& Cogérino, G. (2012). La mise en scène corporelle de l'enseignant d'EPS : les communications non verbales au service de l'efficacité de l'enseignant, STAPS, 98(4), 67-79.

Bonaiuto, M. \& Maricchiolo F. (2007). La comunicazione non verbale. Roma : Carocci Editore.

Bucheton, D. \& Soulé, Y. (2009). Les gestes professionnels et le jeu des postures de l'enseignant dans la classe : un multi-agenda de préoccupations enchâssées. Éducation \& Didactique 3(3), 29-48.

Burel, N. \& Andrieu, B. (2014). Expériences corporelles et gestes professionnels, tome 1, Recherches et Educations, 12.

Cizeron, M. \& Gal-Petitfaux, N. (2005). Connaître la gymnastique et savoir l'enseigner en EP : la nature de la connaissance pédagogique des contenus et son lien à l'instruction en classe, Science et Motricité, 55, 9-33.

Clot, Y. (1999). La fonction psychologique du travail. Paris : Presses Universitaires de France.

Corraze, J. (1980). Les communications non verbales. Paris : Presses Universitaires de France.

Cosnier, J. (1982). Communications et langages gestuels. Les voies du langage, communications verbales, gestuelles et animales. Paris : Dunod.

De Landsheere, G. \& Delchambre, A. (1979). Les comportements non verbaux de l'enseignant. Paris : Nathan.

Delignières, D. (2009). Complexité et compétences : un itinéraire théorique en éducation physique. Paris : Editions Revue EPS.

Dinica, R. C. (2014). Non-verbal Communication-Indispensable Complement of Oral and 


\section{eJRIEPS 37 janvier 2016}

Written Communication, Procedia - Social and behavioral sciences, 137, 105-111.

Durand, M. (2008). Un programme de recherche technologique en formation des adultes. Une approche enactive de l'activité humaine et l'accompagnement de son apprentissage/développement. Education \& Didactique, 2(3), 97-121.

Forest, D. (2008). Agencements didactiques : pour une analyse fonctionnelle du comportement non-ver- bal du professeur. Revue Française de Pédagogie, 165, 77-89.

Fraser, B. J. (2001). Twenty thousand hours : editor's introduction. Learning Environments Research, 4, 1-5.

Gal-Petitfaux, N. (2015). L'activité de l'enseignant d'Education physique chevronné en début de leçon : interactions et engagement intentionnel, eJRIEPS, 34, 63-90.

Gelin, D., Rayou, P. \& Ria, L. (2007). Devenir enseignant. Parcours et Formation. Paris : Armand Colin.

Genevois, G. (1992). Étho-psychologie des communications et pédagogie, Note de synthèse. Revue Française de Pédagogie, 100, 81-103.

Goffman, E. (1973). La mise en scène de la vie quotidienne. Paris : Editions de Minuit.

Hall, E.T. (1971). La dimension cachée. Paris : Editions du Seuil.

Hall, E.T. (1984). Le langage silencieux. Paris : Editions du Seuil.

Kammoun, M.M. \& Amade-Escot, C. (2007). Fonctionnalités didactiques de la gestualité de l'enseignant. Revue EPS, 327, 67-70.

Klinzing, H.G. \& Jackson, I. (1987). Training teachers in nonverbal sensitivity and nonverbal behaviors. International Journal of Educational Research, 2, 589-600.

Laurent, Y. \& Saujat, F. (2014). Place des techniques corporelles dans le travail des enseignants d'EPS et conséquences pour la formation des enseignants expérimentés, Recherches et Educations, 12, 13-24.

Lebreton, D. (2000). Anthropologie du corps et modernité. Paris : PUF.

Leutenegger, F. (2000). Construction d'une « clinique » pour le didactique. Une étude des phénomènes temporels de l'enseignement, Recherche en Didactique des Mathématiques, 20(2), 209-250.

Lisahunter. (2011). Re-embodying (preservice middle years) teachers? An attempt to reposition the body and its presence in teaching and learning, Teaching and teacher education 27(1), 187-200.

Mahut B, (2003). Approche sémiotique des interactions didactiques, geste et verbe en situation didactique, thèse de doctorat, Besançon. 


\section{eJRIEPS 37 janvier 2016}

Mahut B., Mahut N., Gréhaigne, J-F. \& Masselot, M. (2005). Gestuelle de l'enseignant en natation et co-construction du sens. Science \& Motricité, 56, 43-63.

Maturana, H.R. \& Varela, F.J. (1994). L'arbre de la connaissance. Paris : Addison-Wesley France.

Moulin, J-F. (2004). Le discours silencieux du corps enseignant: La communication non verbale du maître dans les pratiques de classe, Carrefours de l'éducation, 17, 142-159.

Pujade-Renaud, C. (1983). Le corps de l'enseignant dans la classe. Paris : ESF.

Ria, L., Saury, J., Sève, C. \& Durand, M. (2001). Les dilemmes des enseignants débutants : Etudes lors des premières expériences de classe en Education Physique. Science et Motricité, 42, 47-58.

Roche, L. \& Gal-Petitfaux, N. (2014). Acquérir des gestes professionnels en EPS grâce à un dispositif de vidéo-formation. Recherches \& Education, 12.

Rovegno, I. (2007). Situated perspective on learning. In D. Kirk, D. Macdonald et M. O'Sullivan (Eds.), The Handbook of Physical Education, (pp. 262-273). London : Sage publications.

Runtz-Christian, E. (2000). Enseignant et comédien, un même métier ?Paris : ESF.

Saury, J., Sève, C., Leblanc, S. \& Durand, M. (2002). Analyse de l'intervention des entraîneurs à l'entraînement et en compétition. Contribution de quatre perspectives de recherche. Science et Motricité, 46, 9-47.

Sperber, D., Wilson, D. (1989). La pertinence. Communication et Cognition. Paris : Les Editions de Minuit.

Suchman, L. (1987). Plans and situated action. The problem of human machine communication. Cambridge : Cambridge University Press.

Tardif, M., Gauthier, C. (2001). L'enseignant comme acteur rationnel. In L. Paquay, M. Altet, E. Charlier \& P. Perrenoud (Eds.), Former des enseignants professionnels : quelles stratégies ? Quelles compétences ? (pp. 231-243). Paris : De Boeck.

Theureau, J. (1992). Le cours d'action: Analyse sémio-logique. Essai d'une anthropologie cognitive située. Berne : Peter Lang.

Theureau, J. (2006). Le cours d'action : Méthode développée. Toulouse : Octarès.

Theureau, J. \& Donin, N. (2005). Comprendre une activité de composition musicale : essai méthodologique sur les relations entre sujet, activité créatrice, environnement et conscience pré-réflexive dans le cadre du programme de recherche cours 


\section{eJRIEPS 37 janvier 2016}

d'action. In J.M. Barbier \& M. Durand (Eds.), Les rapports sujets-activitésenvironnements. Paris : PUF.

Tochon, F. (1993). L'enseignant expert. Paris : Nathan.

Varela, F.J. (1989). Autonomie et connaissance. Essai sur le vivant. Paris : Seuil.

Varela, F.J. (1996).Quel savoir pour l'éthique? Action, sagesse et cognition. Paris : La Découverte.

Varela, F.J., Thompson, E. \& Rosch, E. (1993). L'inscription corporelle de l'esprit. Paris : Seuil.

Vermersch, P. (2000). Approche du singulier. In J.M. Barbier (Ed.), L'analyse de la singularité de l'action (pp. 239-256), Paris : PUF.

Veyrunes, P. (2012). Dynamique de l'activité individuelle et collective en classe lors du «passage dans les rangs ». Revue des Sciences de l'Education, 38(1), 187-208.

Visioli, J. \& Ria, L. (2010). L'expertise des enseignants d'EPS : Quelle prise en compte du contexte et des émotions ? Science et Motricité, 71, 3-19.

Visioli, J., Petiot, O. \& Ria, L. (2014). Le jeu émotionnel des enseignants experts en cours d'Education Physique et Sportive : un moyen de favoriser le plaisir d'enseigner ?. Movement and sport sciences, publié en ligne: http://dx.doi.org/10.1051/sm/2014008.

Vors, O. \& Gal-Petitfaux, N. (2011). Situations de travail par ateliers et configuration de l'activité collective en classe "Réseau Ambition Réussite », eJRIEPS, 22, 96-115.

Vors, O. \& Gal-Petitfaux, N. (2014). Formes d'expériences sensorielles structurant les stratégies d'intervention en classe difficile, Recherches et Educations, 12, 25-42.

Watzlawick, P. (1988). L'invention de la réalité: contributions au constructivisme. Paris : Seuil.

Winkin, Y. (1981). La nouvelle communication. Paris : Seuil.

Zeki, C. P. (2009). The importance of non-verbal communication in classroom management, Procedia - Social and behavioral sciences, 1(1), 1443-1449. 